\title{
Ricci curvature on Alexandrov spaces and rigidity theorems
}

\author{
Hui-Chun Zhang And Xi-Ping Zhu
}

\begin{abstract}
In this paper, we introduce a new notion for lower bounds of Ricci curvature on Alexandrov spaces, and extend Cheeger-Gromoll splitting theorem and Cheng's maximal diameter theorem to Alexandrov spaces under this Ricci curvature condition.
\end{abstract}

\section{Introduction}

Alexandrov spaces with curvature bounded below generalize successfully the concept of lower bounds of sectional curvature from Riemannian manifolds to singular spaces. The seminal paper [4] and the 10th chapter in the text book [3] provide excellent introductions to this field. Many important theorems in Riemannian geometry had been extended to Alexandrov spaces, such as Synge's theorem [30], diameter sphere theorem [28], Toponogov splitting theorem [19], etc.

However, many fundamental results in Riemannian geometry (for example, Bishop-Gromov volume comparison theorem, Cheeger-Gromoll splitting theorem and Cheng's maximal diameter theorem) assume only the lower bounds on Ricci curvature, not on sectional curvature. Therefore, it is a very interesting question how to generalize the concept of lower bounds of Ricci curvature from Riemannian manifolds to singular spaces.

Perhaps the first concept of lower bounds of Ricci curvature on singular spaces was given by Cheeger and Colding (see Appendix 2 in [7]). They, in $[6,7]$, studied Gromov-Hausdorff limit spaces of Riemannian manifolds with Ricci curvature (uniformly) bounded below. Among other results in [6], they proved the following rigidity theorem:

Theorem 1.1 (Cheeger-Colding). Let $M_{i}$ be a sequence of Riemannian manifolds and $M_{i}$ converges to $X$ in sense of Gromov-Hausdorff.

(1) If $X$ contains a line and $\operatorname{Ric}\left(M_{i}\right) \geqslant-\epsilon_{i}$ with $\epsilon_{i} \rightarrow 0$, then $X$ is isometric to a direct product $\mathbb{R} \times Y$ over some length space $Y$. 
(2) If $\operatorname{Ric}\left(M_{i}\right) \geqslant n-1$ and diameter of $M_{i} \operatorname{diam}\left(M_{i}\right) \rightarrow \pi$, then $X$ is isometric to a spherical suspension $[0, \pi] \times \sin Y$ over some length space $Y$.

In [33], Petrunin considered to generalize the lower bounds of Ricci curvature for singular spaces via subharmonic functions.

Recently, in terms of $L^{2}$-Wasserstein space and optimal mass transportation, Sturm [37, 38] and Lott-Villani [16, 17] have given a generalization of "Ricci curvature has lower bounds" for metric measure spaces, ${ }^{1}$ independently. They call that curvature-dimension conditions, denoted by $\operatorname{CD}(n, k)$ with $n \in(1, \infty]$ and $k \in \mathbb{R}$. For the convenience of readers, we repeat their definition of $\operatorname{CD}(n, k)$ in the Appendix of this paper. On the other hand, Sturm in [38] and Ohta in [20] introduced another definition of "Ricci curvature bounded below" for metric measure spaces, the measure contraction property $\operatorname{MCP}(n, k)$, which is a slight modification of a property introduced earlier by Sturm in [39] and in a similar form by Kuwae and Shioya in $[14,15]$. The condition $\operatorname{MCP}(n, k)$ is indeed an infinitesimal version of the Bishop-Gromov relative volume comparison condition. For a metric measure space, Sturm [38] proved that $\operatorname{CD}(n, k)$ implies $\operatorname{MCP}(n, k)$ provided it is nonbranching. ${ }^{2}$ Note that any Alexandrov space with curvature bounded below is non-branching. Recently, Petrunin [31] proved that any $n$-dimensional Alexandrov space with curvature $\geqslant 0$ must satisfy $\operatorname{CD}(n, 0)$ and claimed the general statement that the condition curvature $\geqslant k$ (for some $k \in \mathbb{R}$ ) implies the condition $\mathrm{CD}(n,(n-1) k)$ can be also proved along the same lines.

Let $M$ be a Riemannian manifold with Riemannian distance $d$ and Riemannian volume vol. Lott and Villani in [16] and von Renesse and Sturm in $[36,40]$ proved that $(M, d, \mathrm{vol})$ satisfies $\operatorname{CD}(\infty, k)$ if and only if $\operatorname{Ric}(M) \geqslant k$. Indeed, they proved a stronger weighted version (see Theorem 7.3 in [16] and Theorem 1.1 in [36], Theorem 1.3 in [40]). Let $\phi$ be a smooth function on $M$ with $\int_{M} e^{-\phi} d \mathrm{vol}=1$. Lott and Villani in [17] proved that $\left(M, d, e^{-\phi} \cdot \operatorname{vol}\right)$ satisfies $\operatorname{CD}(n, k)$ if and only if weighted Ricci curvature $\operatorname{Ric}_{n}(M) \geqslant k$ (see Definition 4.20 - the definition of $\mathrm{Ric}_{n}$ - and Theorem 4.22 in [17]). A similar result was proved by Sturm in [38] (see Theorem 1.7 in [38]). In particular, they proved that $(M, d, \mathrm{vol})$ satisfies $\operatorname{CD}(n, k)$ if and only if $\operatorname{Ric}(M) \geqslant k$ and $\operatorname{dim}(M) \leqslant n$. If $\operatorname{dim}(M)=n$, Ohta in [20] and Sturm in [38] proved, independently, that $M$ satisfies $\operatorname{MCP}(n, k)$ is equivalent to $\operatorname{Ric}(M) \geqslant k$.

\footnotetext{
${ }^{1} \mathrm{~A}$ metric measure space is a metric space equipped a Borel measure.

${ }^{2} \mathrm{~A}$ geodesic space is called non-branching if for any quadruple points $z, x_{0}, x_{1}, x_{2}$ with $z$ being the midpoint of $x_{0}$ and $x_{1}$ as well as the midpoint of $x_{0}$ and $x_{2}$, it follows that $x_{1}=x_{2}$.
} 
Nevertheless, since $n$-dimensional norm spaces $\left(V^{n},\|\cdot\|_{p}\right)$ satisfy $\mathrm{CD}(n, 0)$ for every $p>1$ (see, for example, in [41, p. 892]), it is impossible to show Cheeger-Gromoll splitting theorem under $C D(n, 0)$ for general metric measure spaces. Furthermore, it was shown by Ohta in [22] that on a Finsler manifolds $M$, the curvature-dimension condition $\mathrm{CD}(n, k)$ is equivalent to the weighted Finsler Ricci curvature condition $\operatorname{Ric}_{n}(M) \geqslant k$ (see also [23] or [25], refer to [23] for the definition $\mathrm{Ric}_{n}$ in Finsler manifolds). That says, the curvature-dimension condition is somewhat a Finsler geometry character. Seemly, it is difficult to show the rigidity theorems, such as Cheng's maximal diameter theorem and Obata's theorem, under $\operatorname{CD}(n, n-1)$ for general metric measure spaces.

As a compensation, Watanabe [42] proved that if a metric measure space $M$ satisfies $\operatorname{CD}(n, 0)$ or $\operatorname{MCP}(n, 0)$ then $M$ has at most two ends. Ohta [21] proved that a non-branching compact metric measure space with $\operatorname{MCP}(n, n-1)$ and diameter $=\pi$ is homeomorphic to a spherical suspension.

Alexandrov spaces with curvature bounded below have richer geometric information than general metric measure spaces. In particular, a finite dimensional norm space with curvature bounded below must be an innerproduct space. Naturally, one would expect that Cheeger-Gromoll splitting theorem still holds on Alexandrov spaces with suitable non-negative "Ricci curvature condition."

Recently in [12], Kuwae and Shioya proved the following topological splitting theorem for Alexandrov spaces under the $\operatorname{MCP}(n, 0)$ condition:

Theorem 1.2 (Kuwae-Shioya). Let $M^{n}$ be an $n$-dimensional Alexandrov space. Assume that $M^{n}$ contains a line.

(1) If $M$ satisfies $\mathrm{MCP}(n, 0)$, then $M^{n}$ is homeomorphic to a direct product space $\mathbb{R} \times Y$ over some topological space $Y$.

(2) If the singular set of $M^{n}$ is closed and the non-singular set is an (incomplete) $C^{\infty}$ Riemannian manifold of Ric $\geqslant 0$, then $M^{n}$ is isometric to a direct product space $\mathbb{R} \times Y$ over some Alexandrov space $Y$.

We remark that Kuwae and Shioya actually obtained a more general weighted measure version of the above theorem in [13].

In the following, inspired by Petrunin's second variation of arc length [30], we will introduce a new notion of the Ricci curvature bounded below for Alexandrov spaces.

Let $M$ be an $n$-dimensioal Alexandrov space of curvature bounded from below locally without boundary. It is well known in [34] or [32] that, for 
any $p \in M$ and $\xi \in \Sigma_{p}$, there exists a quasi-geodesic starting at $p$ along direction $\xi$. (See [34] or [32, Section 5] for the definition and properties of quasi-geodesics.) According to [30], the exponential map $\exp _{p}: T_{p} \rightarrow M$ is defined as follows. For any $v \in T_{p}$, $\exp _{p}(v)$ is a point on some quasi-geodesic of length $|v|$ starting point $p$ along $v /|v| \in \Sigma_{p}$. If the quasi-geodesic is not unique, we take one of them as the definition of $\exp _{p}(v)$.

Let $\gamma:[0, \ell) \rightarrow M$ be a geodesic. Without loss of generality, we may assume that a neighborhood $U_{\gamma}$ of $\gamma$ has curvature $\geqslant k_{0}$ for some $k_{0}<0$.

According to Section 7 in [4], the tangent cone $T_{\gamma(t)}$ at an interior point $\gamma(t)(t \in(0, \ell))$ can be split into a direct metric product. We denote

$$
\begin{aligned}
& L_{\gamma(t)}=\left\{\xi \in T_{\gamma(t)} \mid \angle\left(\xi, \gamma^{+}(t)\right)=\angle\left(\xi, \gamma^{-}(t)\right)=\pi / 2\right\} \\
& \Lambda_{\gamma(t)}=\left\{\xi \in \Sigma_{\gamma(t)} \mid \angle\left(\xi, \gamma^{+}(t)\right)=\angle\left(\xi, \gamma^{-}(t)\right)=\pi / 2\right\}
\end{aligned}
$$

In [30], Petrunin proved the following second variation formula of arclength.

Proposition 1.1 (Petrunin). Given any two points $q_{1}, q_{2} \in \gamma$, which are not end points, and any positive number sequence $\left\{\varepsilon_{j}\right\}_{j=1}^{\infty}$ with $\varepsilon_{j} \rightarrow 0$, there exists a subsequence $\left\{\widetilde{\varepsilon}_{j}\right\} \subset\left\{\varepsilon_{j}\right\}$ and an isometry $T: L_{q_{1}} \rightarrow L_{q_{2}}$ such that

$$
\begin{aligned}
\left|\exp _{q_{1}}\left(\widetilde{\varepsilon}_{j} u\right), \exp _{q_{2}}\left(\widetilde{\varepsilon}_{j} T v\right)\right| \leqslant & \left|q_{1} q_{2}\right|+\frac{|u v|^{2}}{2\left|q_{1} q_{2}\right|} \cdot \widetilde{\varepsilon}_{j}^{2} \\
& -\frac{k_{0} \cdot\left|q_{1} q_{2}\right|}{6} \cdot\left(|u|^{2}+|v|^{2}+\langle u, v\rangle\right) \cdot \widetilde{\varepsilon}_{j}^{2}+o\left(\widetilde{\varepsilon}_{j}^{2}\right)
\end{aligned}
$$

for any $u, v \in L_{q_{1}}$.

We remark that for a two-dimensional Alexandrov space, Cao et al. in [8] improved the second variation formula such that the above inequality holds for all $\left\{\varepsilon_{j}\right\}_{j=1}^{\infty}$. But for higher dimensions, to the best of our knowledge, we do not know whether the parallel translation $T$ in the above second variation formula can be chosen independent of the sequences $\left\{\varepsilon_{j}\right\}$.

Based on this second variation formula, we can propose a condition which resembles the lower bounds for the radial curvature along the geodesic $\gamma$.

Let $\left\{g_{\gamma(t)}\right\}_{0<t<\ell}$ be a family of functions, where for each $t, g_{\gamma(t)}$ is a continuous function on $\Lambda_{\gamma(t)}$. For simplicity, we call $\left\{g_{\gamma(t)}\right\}_{0<t<\ell}$ to be a continuous function family.

Definition 1.1. A continuous function family $\left\{g_{\gamma(t)}\right\}_{0<t<\ell}$ is said to satisfy Condition $(R C)$, if for any $\epsilon>0$ and any $t_{0} \in(0, \ell)$, there exists a neighborhood $I_{t_{0}}:=\left(t_{0}-\tau^{*}, t_{0}+\tau^{*}\right) \subset(0, \ell)$ with the following property. For any 
two number $s, t \in I_{t_{0}}$ with $s<t$ and for any sequence $\left\{\theta_{j}\right\}_{j=1}^{\infty}$ with $\theta_{j} \rightarrow 0$ as $j \rightarrow \infty$, there exists an isometry $T: \Lambda_{\gamma(t)} \rightarrow \Lambda_{\gamma(s)}$ and a subsequence $\left\{\delta_{j}\right\}$ of $\left\{\theta_{j}\right\}$ such that

$$
\begin{aligned}
& \left|\exp _{\gamma(s)}\left(\delta_{j} l_{1} T \xi\right), \exp _{\gamma(t)}\left(\delta_{j} l_{2} \xi\right)\right| \\
& \quad \leqslant|s-t|+\frac{\left(l_{1}-l_{2}\right)^{2}}{2|s-t|} \cdot \delta_{j}^{2} \\
& \quad \quad-\frac{\left(g_{\gamma(t)}(\xi)-\epsilon\right) \cdot|s-t|}{6} \cdot\left(l_{1}^{2}+l_{1} \cdot l_{2}+l_{2}^{2}\right) \cdot \delta_{j}^{2}+o\left(\delta_{j}^{2}\right)
\end{aligned}
$$

for any $l_{1}, l_{2} \geqslant 0$ and any $\xi \in \Lambda_{\gamma(t)}$.

Let $\mathcal{F}$ denote the set all of continuous function families $\left\{g_{\gamma(t)}\right\}_{0<t<\ell}$, which satisfy Condition $(R C)$.

Clearly, the above proposition shows that $\left\{g_{\gamma(t)}=k_{0}\right\}_{0<t<\ell} \in \mathcal{F}$.

Definition 1.2. We say that $M$ has Ricci curvature bounded below by $(n-1) K$ along $\gamma$, if

$$
\rho:=\sup _{\left\{g_{\gamma(t)}\right\} \in \mathcal{F}} \inf _{0<t<\ell} \oint_{\Lambda_{\gamma(t)}} g_{\gamma(t)}(\xi) \geqslant K,
$$

where $\oint_{\Lambda_{x}} g_{x}(\xi)=\frac{1}{\operatorname{vol}\left(\Lambda_{x}\right)} \int_{\Lambda_{x}} g_{x}(\xi) d \xi$.

We say $M$ has Ricci curvature bounded below by $(n-1) K$ on an open set $U \subset M$, if for each point $p \in U$, there is a neighborhood $U_{p}$ of $p$ with $U_{p} \subset U$ such that $M$ has Ricci curvature bounded below by $(n-1) K$ along every geodesic $\gamma:[0, \ell) \rightarrow U_{p}$. When $U=M$, we say $M$ has Ricci curvature bounded below by $(n-1) K$ and denote $\operatorname{Ric}(M) \geqslant(n-1) K$.

Remark 1.1. (i) When $M$ is a smooth Riemannian manifold, by the second variation of formula of arc-length, it is easy to see Condition $(\mathrm{RC})$ is equivalent to

$$
\sec _{M}\left(\Pi_{t}\right) \geqslant g_{\gamma(t)}(\xi)
$$

where $\Pi_{t} \subset T_{\gamma(t)}$ is any two-dimensional subspace, spanned by $\gamma^{\prime}(t)$ and a $\xi \in \Lambda_{\gamma(t)}$. Thus in a Riemannian manifold, our definition on Ricci curvature bounded below by $(n-1) K$ is exactly the classical one.

(ii) Let $M$ be an $n$-dimensional Alexandrov space with curvature $\geqslant K$. The above Proposition 1.1 shows that $\operatorname{Ric}(M) \geqslant(n-1) K$. 
(iii) Recall that Petrunin in [31] proved any $n$-dimensional Alexandrov space $M$ with curvature $\geqslant K$ must satisfy the curvature-dimension condition $\mathrm{CD}(n,(n-1) K)$. In the Appendix, by modifying Petrunin's proof in [31], we will show that any $n$-dimensional Alexandrov space $M$ with $\operatorname{Ric}(M) \geqslant(n-1) K$ also satisfies $\operatorname{CD}(n,(n-1) K)$.

(iv) At the present stage, we do not know if the Ricci curvature condition $\operatorname{Ric}(M) \geqslant(n-1) K$ is equivalent to the curvature-dimension condition $\mathrm{CD}(n,(n-1) K)$. We will investigate this question in future.

Our main results in this paper are the following splitting theorem and maximal diameter theorem.

Theorem 1.3 (Splitting theorem). Let $M$ be an $n$-dimensional complete non-compact Alexandrov space with non-negative Ricci curvature and $\partial M=$ $\varnothing$. If $M$ contains a line, then $M$ is isometric to a direct metric product $\mathbb{R} \times N$ for some Alexandrov space $N$ with non-negative Ricci curvature.

Theorem 1.4 (Maximal diameter theorem). Let $M$ be an $n$-dimensional compact Alexandrov space with Ricci curvature bounded below by $n-1$ and $\partial M=\varnothing$. If the diameter of $M$ is $\pi$, then $M$ is isometric to a spherical suspension over an Alexandrov space with curvature $\geqslant 1$.

An open question for the curvature-dimension condition $\operatorname{CD}(n, k)(k \neq 0)$ is "from local to global" (See, for example, the 30th chapter in [41]). In particular, given a metric measure space which admits a covering and satisfies $\mathrm{CD}(n, k)(k \neq 0)$, we do not know if the covering space with pullback metric still satisfies $\mathrm{CD}(n, k)$.

One advantage of our definition of the Ricci curvature bounded below on Alexandrov spaces is that the definition is purely local. In particular, any covering space of an $n$-dimensional Alexandrov space with Ricci curvature bounded below by $(n-1) K$ still satisfies the condition Ric $\geqslant(n-1) K$. Meanwhile, we note that Bishop-Gromov volume comparison theorem also holds on an Alexandrov space with Ricci curvature bounded below (see Corollary A.1 in the Appendix). Consequently, the same proofs as in Riemannian manifold case (see [1] and, for example,[27, pp. 275-276]) give the following estimates on the fundamental group and the first Betti number.

Corollary 1.1. Let $M$ be a compact $n$-dimensional Alexandrov space with non-negative Ricci curvature and $\partial M=\varnothing$. Then its fundamental group has a finite index Bieberbach subgroup. 
Corollary 1.2. Let $M$ be an n-dimensional Alexandrov space with nonnegative Ricci curvature and $\partial M=\varnothing$. Then any finitely generated subgroup of $\pi_{1}(M)$ has polynomial growth of degree $\leqslant n$. If some finitely generated subgroup of $\pi_{1}(M)$ has polynomial growth of degree $=n$, then $M$ is compact and flat.

Corollary 1.3. Let $M$ be an $n$-dimensional Alexandrov space with $\partial M=\varnothing$.

(1) If $\operatorname{Ric}(M) \geqslant(n-1) K>0$, then its fundamental group is finite.

(2) If $\operatorname{Ric}(M) \geqslant(n-1) K$ and diameter of $M \leqslant D$, then

$$
b_{1}(M) \leqslant C\left(n, K^{2} \cdot D\right)
$$

for some function $C\left(n, K^{2} \cdot D\right)$.

Moreover, there exists a constants $\kappa(n)>0$ such that if $K^{2} \cdot D \geqslant-\kappa(n)$, then $b_{1}(M) \leqslant n$.

The paper is organized as follows. In Section 2, we recall some necessary materials for Alexandrov spaces. In Section 3, we will define a new representation of Laplacian along a geodesic and will prove the comparison theorem for the newly defined representation of Laplacian (see Theorem 3.1). In Section 4 , we will discuss the rigidity part of the comparison theorem. The maximal diameter theorem and the splitting theorem will be proved in Sections 5 and 6 , respectively. In the Appendix, we give a modification of Petrunin's proof in [31] to show that the condition on Ricci curvature bounded below implies the curvature-dimension condition (see Proposition A.1).

\section{Preliminaries}

A metric space $(X,|\cdot, \cdot|)$ is called a length space if for any two point $p, q \in X$, the distance between $p$ and $q$ is given by

$$
|p q|=\inf _{\gamma, \gamma \text { connect } p, q} \operatorname{Length}(\gamma)
$$

A length space $X$ is called a geodesic space if for any two point $p, q \in X$, there exists a curve $\gamma$ connecting $p$ and $q$ such that $\operatorname{Length}(\gamma)=|p q|$. Such a curve is called a shortest curve. A geodesic is a unit-speed shortest curve. 
Recall that a length space $X$ has curvature $\geqslant k$ in an open set $U \subset X$ if for any quadruple $(p ; a, b, c) \subset U$, there holds

$$
\widetilde{\angle}_{k} a p b+\widetilde{\angle}_{k} b p c+\widetilde{\angle}_{k} c p a \leqslant 2 \pi,
$$

where $\widetilde{\angle}_{k} a p b, \widetilde{L}_{k} b p c$ and $\widetilde{L}_{k} c p a$ are the comparison angles in the $k$-plane. A length space $M$ is called an Alexandrov space with curvature bounded from below locally (for short, we say $M$ to be an Alexandrov space), if it is locally compact and any point in $M$ has an open neighborhood $U \subset M$ such that $M$ has curvature $\geqslant k_{U}$ in $U$, for some $k_{U} \in \mathbb{R}$.

Let $M$ be an Alexandrov space without boundary and $U \subset M$ be an open set. A locally Lipschitz function $u$ on $U$ is said to be $\lambda$-concave on $U$ if for any geodesic $\gamma \subset U$, the one-variable function

$$
u \circ \gamma(t)-\lambda t^{2} / 2
$$

is concave. A function $u$ on $M$ is said to be semi-concave if for any point $x \in M$ there is a neighborhood $U_{x} \ni x$ and a real number $\lambda_{x}$ such that the restriction $\left.u\right|_{U_{x}}$ is $\lambda_{x}$-concave.

Let $\psi: \mathbb{R} \rightarrow \mathbb{R}$ be a continuous function. A function $u$ on $M$ called $\psi(u)$ - concave if for any point $x \in M$ and any $\varepsilon>0$ there is a neighborhood $U_{x} \ni x$ such that $\left.u\right|_{U_{x}}$ is $(\psi \circ u(x)+\varepsilon)$-concave.

If $M$ has curvature $\geqslant k$ in $U$, then it is well known that the function $u=\varrho_{k} \circ \operatorname{dist}_{p}$ is $(1-k u)$-concave in $U \backslash\{p\}$, where

$$
\varrho_{k}(v)= \begin{cases}\frac{1}{k}(1-\cos (\sqrt{k} v)) & \text { if } \quad k>0 \\ \frac{v^{2}}{2} & \text { if } \quad k=0 \\ \frac{1}{k}(\cosh (\sqrt{-k} v)-1) & \text { if } \quad k<0\end{cases}
$$

(see, for example,[32, Section 1]).

Let $u$ be a semi-concave function on $M$. For any point $p \in M$, there exists a $u$-gradient curve starting at $p$. Hence $u$ generates a gradient flow $\Phi_{u}^{t}: M \rightarrow M$, which is a locally Lipschitz map. (Actually, it is just a semiflow, because backward flow $\Phi_{u}^{-t}$ is not always well-defined.) Particularly, if $u$ is concave, the gradient flow is a 1-Lipschitz map. We refer to Sections 1 and 2 in [32] for the details on semi-concave functions, gradient curves and gradient flows. 


\section{Laplacian comparison theorem}

Let $M$ be an $n$-dimensional Alexandrov space without boundary. A canonical Dirichlet form $\mathcal{E}$ is defined by

$$
\mathcal{E}(u, v):=\int_{M}\langle\nabla u, \nabla v\rangle d \mathrm{vol}, \quad \text { for } \quad u, v \in W_{0}^{1,2}(M) .
$$

(see [11]). The Laplacian associated to the canonical Dirichlet form is given as follows. Let $u: U \subset M \rightarrow \mathbb{R}$ be a $\lambda$-concave function. The (canonical) Lapliacian of $u$ as a sign-Radon measure is defined by

$$
\int_{M} \phi d \Delta u=-\mathcal{E}(u, \phi)=-\int_{M}\langle\nabla \phi, \nabla u\rangle d \mathrm{vol}
$$

for all Lipschitz function $\phi$ with compact support in $U$. In [31], Petrunin proved

$$
\Delta u \leqslant n \lambda \cdot \operatorname{vol}
$$

in particular, the singular part of $\Delta u$ is non-positive. If $M$ has curvature $\geqslant K$, then any distance function $\operatorname{dist}_{p}(x):=d(p, x)$ is $\cot _{K}$ odist $_{p}$-concave on $M \backslash\{p\}$, where the function $\cot _{K}(s)$ is defined by

$$
\cot _{K}(s)= \begin{cases}\frac{\sqrt{K} \cdot \cos (\sqrt{K} s)}{\sin (\sqrt{K} s)} & \text { if } \quad K>0, \\ \frac{1}{s} & \text { if } \quad K=0, \\ \frac{\sqrt{-K} \cdot \cosh (\sqrt{-K} s)}{\sinh (\sqrt{-K} s)} & \text { if } \quad K<0 .\end{cases}
$$

It is a solution of the ordinary differential equation $\chi^{\prime}(s)=-K-\chi^{2}(s)$. Therefore the above inequality $\Delta u \leqslant n \lambda \cdot$ vol gives a Laplacian comparison theorem for the distance function on Alexandrov spaces.

In [12], by using the $D C$-structure (see [29]), Kuwae-Shioya defined a distributional Laplacian for a distance function dist $_{p}$ by

$$
\Delta \operatorname{dist}_{p}=D_{i}\left(\sqrt{\operatorname{det}\left(g_{i j}\right)} g^{i j} \partial_{j} \operatorname{dist}_{p}\right)
$$

on a local chart of $M \backslash \mathcal{S}_{\epsilon}$ for sufficiently small positive number $\epsilon$, where

$$
\mathcal{S}_{\epsilon}:=\left\{x \in M: \operatorname{vol}\left(\Sigma_{x}\right) \leqslant \operatorname{vol}\left(\mathbb{S}^{n-1}\right)-\epsilon\right\}
$$


and $D_{i}$ is the distributional derivative. Note that the union of all $\mathcal{S}_{\epsilon}$ has zero measure. One can view the distributional Laplacian $\Delta \operatorname{dist}_{p}$ as a signRadon measure. In [11], Kuwae, Machigashira and Shioya proved that the distributional Laplacian is actually a representation of the previous (canonical) Laplacian on $M \backslash \mathcal{S}_{\epsilon}$. Moreover in [12], Kuwae and Shioya extended the Laplacian comparison theorem under the weaker condition $\mathrm{BG}(k)$.

Both of the above canonical Laplacian and its DC representation (i.e., the distributional Laplacian) make sense up to a set which has zero measure.

In Riemannian geometry, according to Calabi, the Laplacian comparison theorem holds in barrier sense, not just in distribution sense. In this section, we will try to give a new representation of the above canonical Laplacian of a distance function, which makes sense in $W_{p}$, the set of points $z \in M$ such that the geodesic $p z$ can be extended beyond $z$. We will also prove a comparison theorem for the new representation under our Ricci curvature condition.

Let $M$ denote an $n$-dimensional complete Alexandrov space without boundary. Fix a geodesic $\gamma:[0, \ell) \rightarrow M$ with $\gamma(0)=p$ and denote $f=\operatorname{dist}_{p}$. Let $x \in \gamma \backslash\{p\}$ and $L_{x}, \Lambda_{x}$ be as above in Section 1. Clearly, we may assume that $M$ has curvature $\geqslant k_{0}$ (for some $k_{0}<0$ ) in a neighborhood $U_{\gamma}$ of $\gamma$.

Perelman in [29] defined a Hessian for a semi-concave function $u$ on almost all point $x \in M$, denoted by $\operatorname{Hess}_{x} u$. It is a bi-linear form on $T_{x}(=$ $\mathbb{R}^{n}$ ). But for the given geodesic $\gamma$, we cannot insure that the Hessian is well defined along $\gamma$.

We now define a version of Hessian and Laplacian for the distance function $f$ along the geodesic $\gamma$ as follows. Note that the tangent space at an interior point $x \in \gamma$ can be split to $L_{x} \times \mathbb{R}$ and $f \circ \gamma$ is linear. So we only need to define the Hessian on the set of orthogonal directions $\Lambda_{x}$.

Throughout this paper, $\mathcal{S}$ will always denote the set of all sequences $\left\{\theta_{j}\right\}_{j=1}^{\infty}$ with $\theta_{j} \rightarrow 0$ as $j \rightarrow \infty$ and $\theta_{j+1} \leqslant \theta_{j}$.

Definition 3.1. Let $x \in \gamma \backslash\{p\}$. Given a sequence $\theta:=\left\{\theta_{j}\right\}_{j=1}^{\infty} \in \mathcal{S}$, we define a function $H_{x}^{\theta} f: \Lambda_{x} \rightarrow \mathbb{R}$ by

$$
H_{x}^{\theta} f(\xi) \stackrel{\text { def }}{=} \limsup _{s \rightarrow 0, s \in \theta} \frac{f \circ \exp _{x}(s \cdot \xi)-f(x)}{s^{2} / 2}
$$

and

$$
\Delta^{\theta} f(x) \stackrel{\text { def }}{=}(n-1) \cdot \oint_{\Lambda_{x}} H_{x}^{\theta} f(\xi)
$$


Since $U_{\gamma}$ has curvature $\geqslant k_{0}$, we know that $f$ is $\cot _{k_{0}}(|p x|)$-concave and $\operatorname{dist}_{\gamma(\ell)}$ is $\cot _{k_{0}}(|x \gamma(\ell)|)$-concave near $x$, which imply

$$
H_{x}^{\theta} f \leqslant \cot _{k_{0}}(|p x|)
$$

for any sequence $\theta \in \mathcal{S}$, and

$$
\left|\gamma(\ell) \exp _{x}(s \cdot \xi)\right| \leqslant|x \gamma(\ell)|+\cot _{k_{0}}(|x \gamma(\ell)|) \cdot s^{2} / 2+o\left(s^{2}\right)
$$

for any $\xi \in \Lambda_{x}$. Then by triangle inequality, we have

$$
H_{x}^{\theta} f \geqslant-\cot _{k_{0}}(|x \gamma(\ell)|)
$$

Thus $H_{x}^{\theta} f$ is well defined and bounded. It is easy to see that $H_{x}^{\theta} f$ is measurable on $\Lambda_{x}$ and thus it is integrable.

If there exists Perelman's Hessian of $f$ at a point $x$ (see [29]), then $H_{x}^{\theta} f(\xi)=\operatorname{Hess}_{x} f(\xi, \xi)$ for all $\xi \in \Lambda_{x}$ and $\theta \in \mathcal{S}$.

Denote by $\operatorname{Reg}_{f}$ the set of points $z \in M$ such that there exists Perelman's Hessian of $f$ at $z$. If we write the Lebesgue decomposition of the canonical Laplacian $\Delta f=(\Delta f)^{\text {sing }}+(\Delta f)^{\text {ac }} \cdot$ vol, with respect to the $n$-dimension Hausdorff measure vol, then $(\Delta f)^{\mathrm{ac}}(x)=\operatorname{Tr} \operatorname{Hess}_{x} f=\Delta^{\theta} f(x)$ for all $x \in W_{p} \cap \operatorname{Reg}_{f}$ and $\theta \in \mathcal{S}$. It was shown in $[24,29]$ that $\operatorname{Reg}_{f} \cap W_{p}$ has full measure in $M$. Thus $\Delta^{\theta} f(x)$ is actually a representation of the absolutely continuous part of the canonical Laplacian $\Delta f$ on $W_{p}$.

Note from the definition that if $\theta_{1} \subset \theta_{2}$, then

$$
H_{x}^{\theta_{1}} f \leqslant H_{x}^{\theta_{2}} f \text { and } \Delta^{\theta_{1}} f(x) \leqslant \Delta^{\theta_{2}} f(x)
$$

The following lemma is a discrete version of the propagation equation of the Hessian of $f$ along the geodesic $\gamma$.

Lemma 3.1. Let $f=\operatorname{dist}_{p}$. Given $\epsilon>0$, a continuous functions family $\left\{g_{\gamma(t)}\right\}_{0<t<\ell}$ and a sequence $\left\{\theta_{j}\right\}_{j=1}^{\infty} \in \mathcal{S}$. Let $y, z \in \gamma$ with $|p y|<|p z|$. We assume that a isometry $T: \Lambda_{z} \rightarrow \Lambda_{y}$ and the subsequence $\delta:=\left\{\delta_{j}\right\} \subset\left\{\theta_{j}\right\}$ such that (1.1) holds. Then

$$
H_{z}^{\delta} f(\xi) \leqslant l^{2} \cdot H_{y}^{\delta} f(\eta)+\frac{(l-1)^{2}}{|y z|}-\frac{l^{2}+l+1}{3} \cdot|y z| \cdot\left(g_{z}(\xi)-\epsilon\right)
$$

for any $l \geqslant 0$ and any $\xi \in \Lambda_{z}, \eta=T \xi \in \Lambda_{y}$. 
Proof. For any $\xi \in \Lambda_{z}$, we can choose a subsequence $\left\{\delta_{j}^{\prime}\right\} \subset\left\{\delta_{j}\right\}$ such that

$$
H_{z}^{\delta} f(\xi)=\lim _{j \rightarrow \infty} \frac{f\left(\exp _{z}\left(\delta_{j}^{\prime} \xi\right)\right)-f(z)}{\delta_{j}^{\prime 2} / 2}
$$

Then, we have

$$
f\left(\exp _{z}\left(\delta_{j}^{\prime} \xi\right)\right)=f(z)+\frac{\delta_{j}^{\prime 2}}{2} H_{z}^{\delta} f(\xi)+o\left(\delta_{j}^{\prime 2}\right)
$$

for any $l \geqslant 0$. By definition, we have

$$
f\left(\exp _{y}\left(\delta_{j}^{\prime} \cdot l \eta\right)\right) \leqslant f(y)+\frac{\left(l \delta_{j}^{\prime}\right)^{2}}{2} H_{y}^{\delta} f(\eta)+o\left(\delta_{j}^{\prime 2}\right) .
$$

Note that

$$
f(z)-f(y)=|y z|
$$

and

$$
f\left(\exp _{z}\left(\delta_{j}^{\prime} \xi\right)\right)-f\left(\exp _{y}\left(\delta_{j}^{\prime} \cdot l \eta\right)\right) \leqslant\left|\exp _{z}\left(\delta_{j}^{\prime} \xi\right), \exp _{y}\left(\delta_{j}^{\prime} \cdot l \eta\right)\right|
$$

By combining (3.4)-(3.7) and using (1.1) with $l_{1}=l, l_{2}=1$, we have

$$
\begin{aligned}
& \frac{\delta_{j}^{\prime 2}}{2}\left(H_{z}^{\delta} f(\xi)-l^{2} \cdot H_{y}^{\delta} f(\eta)\right)+o\left(\delta_{j}^{\prime 2}\right) \\
& \quad \leqslant \delta_{j}^{\prime 2} \cdot\left(\frac{(l-1)^{2}}{2|y z|}-\frac{g_{r}(\xi)-\epsilon}{6} \cdot|y z| \cdot\left(l^{2}+l+1\right)\right)+o\left(\delta_{j}^{\prime 2}\right),
\end{aligned}
$$

for any $l \geqslant 0$. Hence

$$
H_{z}^{\delta} f(\xi)-l^{2} \cdot H_{y}^{\delta} f(\eta) \leqslant \frac{(l-1)^{2}}{|y z|}-\frac{l^{2}+l+1}{3} \cdot|y z| \cdot\left(g_{z}-\epsilon\right) .
$$

This completes the proof of the lemma.

The following result is the comparison for the above defined representation of Laplacian.

Theorem 3.1. Let $f=\operatorname{dist}_{p}$ and $x \in \gamma \backslash\{p\}$. If $M$ has Ricci $\geqslant(n-1) K$ along the geodesic $\gamma(t)$, then, given any sequence $\left\{\theta_{j}\right\}_{j=1}^{\infty} \in \mathcal{S}$, there exists a 
subsequence $\delta=\left\{\delta_{j}\right\}$ of $\left\{\theta_{j}\right\}$ such that

$$
\Delta^{\delta} f(x) \leqslant(n-1) \cdot \cot _{K}(|p x|) .
$$

(If $K>0$, we add assumption $|p x|<\pi / \sqrt{K}$.)

Proof. Arbitrarily fix two constants $\epsilon>0$ and $K^{\prime}<K$ with $10 \epsilon<K-K^{\prime}$.

We can choose a point $y \in p x$ such that $|p y|>\epsilon$ and

$$
\cot _{k_{0}}(|p y|) \leqslant \cot _{K^{\prime}}(|p y|-\epsilon) .
$$

By our definition of Ricci curvature $\geqslant(n-1) K$ along $\gamma$, there exists a continuous function family $\left\{g_{\gamma(t)}\right\}_{0<t<\ell} \in \mathcal{F}$ such that

$$
\oint_{\Lambda_{\gamma(t)}} g_{\gamma(t)} \geqslant K-\epsilon, \quad \forall t \in(0, \ell) .
$$

We take a sufficiently small number $\omega>0$.

For any $t_{0} \in[|p y|,|p x|]$, there is a neighborhood $I_{t_{0}}$ coming from Condition (RC) such that $\left|I_{t_{0}}\right|<\omega$. All of these neighborhoods form an open covering of $[|p y|,|p x|]$. Let $I_{1}, I_{2}, \ldots, I_{N}$ be a finite sub-covering of $[|p y|,|p x|]$. We take $x_{a} \in I_{a} \cap I_{a+1}$ for all $1 \leqslant a \leqslant N-1$ and set $y=x_{0}, x=x_{N}$. We can assume that $\left|p x_{a}\right|<\left|p x_{a+1}\right|$ for all $0 \leqslant a \leqslant N-1$.

By Condition (RC), we can find a subsequence $\left\{\delta_{1, j}\right\} \subset\left\{\theta_{j}\right\}$ and an isometry $T_{1}: \Lambda_{x_{1}} \rightarrow \Lambda_{x_{0}}$ such that (1.1) holds. Next, we can find a further subsequence $\left\{\delta_{2, j}\right\} \subset\left\{\delta_{1, j}\right\}$ and an isometry $T_{2}: \Lambda_{x_{2}} \rightarrow \Lambda_{x_{1}}$ such that (1.1) holds. After a finite step of these procedures, we get a subsequence $\delta=\left\{\delta_{j}\right\} \subset\left\{\delta_{N-1, j}\right\} \subset \cdots \subset\left\{\theta_{j}\right\}$ and a family isometries $\left\{T_{a+1}\right\}_{a=0}^{N-1}, T_{a+1}$ : $\Lambda_{x_{a+1}} \rightarrow \Lambda_{x_{a}}$ such that, for each $a=0,1, \ldots, N-1$,

$$
\begin{aligned}
& \left|\exp _{x_{a}}\left(\delta_{j} l_{1} T_{a+1} \xi\right), \exp _{x_{a+1}}\left(\delta_{j} l_{2} \xi\right)\right| \\
& \leqslant\left|x_{a} x_{a+1}\right|+\frac{\left(l_{1}-l_{2}\right)^{2}}{2 \cdot\left|x_{a} x_{a+1}\right|} \cdot \delta_{j}^{2} \\
& \quad \quad-\frac{\left(g_{t}(\xi)-\epsilon\right) \cdot\left|x_{a} x_{a+1}\right|}{6} \cdot\left(l_{1}^{2}+l_{1} \cdot l_{2}+l_{2}^{2}\right) \cdot \delta_{j}^{2}+o\left(\delta_{j}^{2}\right)
\end{aligned}
$$

for any $l_{1}, l_{2} \geqslant 0$ and any $\xi \in \Lambda_{x_{a+1}}$.

Claim: For all $0 \leqslant a \leqslant N-1$, we have

$$
\oint_{\Lambda_{x_{a}}} H_{x_{a}}^{\delta} f \leqslant \cot _{K^{\prime}}\left(\left|p x_{a}\right|-\epsilon\right),
$$

as $\omega$ is sufficiently small. 
We will prove the claim by induction argument with respect to $a$.

Firstly, we know from (3.8) that the case $a=0$ is held.

Set $q=x_{a}, r=x_{a+1}, \mu=\left|x_{a} x_{a+1}\right|$ and $T=T_{a+1}$. Now we suppose that the claim is held for the case $a$, i.e.,

$$
\oint_{\Lambda_{q}} H_{q}^{\delta} f \leqslant \cot _{K^{\prime}}(|p q|-\epsilon)
$$

We need to show the claim is also held for the case $a+1$.

Consider the functions on $\Lambda_{r}$

$$
F_{l}(\xi)=l^{2} \cdot H_{q}^{\delta} f(T(\xi))+\frac{(l-1)^{2}}{\mu}-\frac{l^{2}+l+1}{3} \cdot \mu \cdot\left(g_{r}(\xi)-\epsilon\right) .
$$

From Lemma 3.1 above, we have

$$
H_{r}^{\delta} f \leqslant F_{l}
$$

for any $l \geqslant 0$.

On the other hand, from (3.9),

$$
\begin{aligned}
\oint_{\Lambda_{r}} F_{l} & =l^{2} \cdot \oint_{\Lambda_{r}} H_{q}^{\delta} f \circ T+\frac{(l-1)^{2}}{\mu}-\frac{l^{2}+l+1}{3} \cdot \mu \cdot\left(\oint_{\Lambda_{r}} g_{r}(\xi)-\epsilon\right) \\
11) & \leqslant l^{2} \cdot\left(\cot _{K}(|p q|-\epsilon)\right)+\frac{(l-1)^{2}}{\mu}-\frac{l^{2}+l+1}{3} \cdot \mu \cdot \bar{K}
\end{aligned}
$$

for any $l \geqslant 0$, where $\bar{K}=K-2 \epsilon$.

By setting

$$
C_{1}=\max _{|p y| \leqslant t \leqslant|p x|}\left|\cot _{K^{\prime}}^{\prime \prime}(t-\epsilon)\right|,
$$

we have

(3.12) $\cot _{K^{\prime}}(|p q|-\epsilon) \leqslant \cot _{K^{\prime}}(|p r|-\epsilon)+\mu\left(K^{\prime}+\cot _{K^{\prime}}^{2}(|p q|-\epsilon)\right)+C_{1} \mu^{2}$.

Thus by combining (3.11) and (3.12), we get

$$
\oint_{\Lambda_{r}} F_{l} \leqslant \cot _{K^{\prime}}(|p r|-\epsilon)+A_{\mu}(l)
$$


where

$$
\begin{aligned}
A_{\mu}(l)= & \mu\left(K^{\prime}+\cot _{K^{\prime}}^{2}(|p q|-\epsilon)\right)+C_{1} \mu^{2}+\left(l^{2}-1\right) \cot _{K^{\prime}}(|p q|-\epsilon) \\
& +\frac{(l-1)^{2}}{\mu}-\frac{l^{2}+l+1}{3} \cdot \mu \cdot \bar{K}
\end{aligned}
$$

Denote by $B=1 / \mu-\mu \bar{K} / 3$ and $\cot =\cot _{K^{\prime}}(|p q|-\epsilon)$. Note that

$$
\cot _{K^{\prime}}(|p x|-\epsilon) \leqslant \cot \leqslant \cot _{K^{\prime}}(|p y|-\epsilon) .
$$

Since $\omega$ is small and $\mu \leqslant \omega$, we can assume that $\cot +B>0$. Choose $\tilde{l}=$ $-(B+\mu \bar{K} / 2) /(\cot +B)$. Then we get

$$
\begin{aligned}
A_{\mu}(\widetilde{l}) & =\frac{-(B+\mu \bar{K} / 2)^{2}+\left(\mu\left(K^{\prime}+\cot ^{2}\right)+C_{1} \mu^{2}-\cot +B\right) \cdot(\cot +B)}{\cot +B} \\
& \leqslant \frac{K^{\prime}-\bar{K}+C_{2} \mu+C_{3} \mu^{2}}{\cot +B},
\end{aligned}
$$

where $C_{2}, C_{3}$ are positive constants independent of $\mu, \omega$ (may be depending on $\epsilon, K^{\prime}, x$ and $\left.y\right)$. Using $\mu \leqslant \omega$, we get

$$
A_{\mu}(\widetilde{l}) \leqslant \frac{K^{\prime}-\bar{K}+C_{2} \omega+C_{3} \omega^{2}}{\cot +B} \leqslant 0
$$

as $\omega$ is sufficiently small. Hence, by combining $(3.10),(3.13)$ and $A_{\mu}(\widetilde{l}) \leqslant 0$, we get

$$
\oint_{\Lambda_{r}} H_{r}^{\delta} f \leqslant \oint_{\Lambda_{r}} F(\widetilde{l}) \leqslant \cot _{K^{\prime}}(|p r|-\epsilon) .
$$

This completes the proof of the claim. In particular, we have

$$
\oint_{\Lambda_{x}} H_{x}^{\delta} f \leqslant \cot _{K^{\prime}}(|p x|-\epsilon) .
$$

Thus by the arbitrariness of $\epsilon$ and $K^{\prime}$ and a standard diagonal argument, we obtain a subsequence of $\delta$, denoted again by $\delta$, such that

$$
\Delta^{\delta} f(x) \leqslant(n-1) \cdot \cot _{K}(|p x|)
$$

Therefore, we have completed the proof of the theorem. 


\section{Rigidity estimates}

We continue to consider an $n$-dimensional complete Alexandrov space $M$ without boundary. Fix a geodesic $\gamma:[0, \ell) \rightarrow M$ with $\gamma(0)=p$ and denote $f=\operatorname{dist}_{p}$.

Let $x \in \gamma \backslash\{p\}$ and $L_{x}, \Lambda_{x}$ be as above. We still assume that a neighborhood $U_{\gamma}$ of $\gamma$ has curvature $\geqslant k_{0}$ (for some constant $k_{0}<0$ ).

Lemma 4.1. Assume $M$ has Ricci $\geqslant(n-1) K$ along the geodesic $\gamma(t)$. Let $f=\operatorname{dist}_{p}$ and $x$ be an interior point on the geodesic $\gamma(t)$. Given a sequence $\theta=\left\{\theta_{j}\right\}_{j=1}^{\infty} \in \mathcal{S}$, if

$$
\Delta^{\theta^{\prime}} f(x)=(n-1) \cdot \cot _{K}(|p x|)
$$

for any subsequence $\theta^{\prime}=\left\{\theta_{j}^{\prime}\right\}$ of $\theta$, then there exists a subsequence $\delta=\left\{\delta_{j}\right\}$ of $\theta$ such that

$$
H_{x}^{\delta} f(\xi)=\cot _{K}(|p x|)
$$

almost everywhere $\xi \in \Lambda_{x}$.

(If $K>0$, we add assumption $|p x|<\pi / \sqrt{K}$.)

Proof. At first, we will prove the following claim:

Claim. For any $\epsilon>0$, we can find a subsequence $\left\{\delta_{j}\right\}$ of $\theta$ and an integrable function $h$ on $\Lambda_{x}$ such that

$$
H_{x}^{\delta} f \leqslant h \quad \text { and } \quad \oint_{\Lambda_{x}}\left(h-\cot _{K}(|p x|)\right)^{2} \leqslant\left(3+2\left|\cot _{K}(|p x|)\right|\right) \epsilon
$$

By our definition of Ricci curvature $\geqslant(n-1) K$ along $\gamma$, there exists a continuous function family $\left\{g_{\gamma(t)}\right\}_{0<t<\ell} \in \mathcal{F}$ such that

$$
\oint_{\Lambda_{\gamma(t)}} g_{\gamma(t)} \geqslant K-\epsilon, \quad \forall t \in(0, \ell)
$$

We may assume $g_{x} \geqslant k_{0}$, otherwise, we replace it by $\max \left\{g_{x}, k_{0}\right\}$.

By the definition of Condition (RC), we have a neighborhood $I(\subset(0, \ell))$ of $\gamma^{-1}(x)$ such that for arbitrarily taking a point $w \in \gamma(I)$ with $|p w|<$ $|p x|$, there exists a subsequence $\widetilde{\delta}=\left\{\widetilde{\delta}_{j}\right\}$ of $\theta$ and an isometric $T: \Lambda_{x} \rightarrow \Lambda_{w}$ 
such that (1.1) holds. By using Lemma 3.1 and choosing $l=1$, we have

$$
\left(g_{x}-\epsilon\right) \cdot|x w| \leqslant H_{w}^{\widetilde{\delta}} f-H_{x}^{\widetilde{\delta}} f .
$$

By (3.2) and the fact that $f$ is $\cot _{k_{0}}(|p \cdot|)$-concave, we have

$$
H_{x}^{\widetilde{\delta}} f \geqslant-\cot _{k_{0}}(|x \gamma(\ell)|) \quad \text { and } \quad H_{w}^{\widetilde{\delta}} f \leqslant \cot _{k_{0}}(|p w|) .
$$

Thus by combining these with (4.3) and the fact $g_{x} \geqslant k_{0}$, we get

$$
\left|g_{x}\right| \leqslant C_{4}
$$

for some constant $C_{4}$, which may depend on $\epsilon, x$ and $|I|$.

Choose a point $z \in \gamma(I)$ with $|p x| / 2<|p z|<|p x|$ and $|x z| \ll \min \{\epsilon,|I|\}$. Then, by Condition (RC), there exists a subsequence $\left\{\delta_{j}^{\prime}\right\}$ of $\theta$ and an isometry $T: \Lambda_{x} \rightarrow \Lambda_{z}$ satisfying (1.1). From Theorem 3.1, we can find a subsequence $\left\{\delta_{j}\right\} \subset\left\{\delta_{j}^{\prime}\right\}$ such that

$$
\Delta^{\delta} f(z) \leqslant(n-1) \cdot \cot _{K}(|p z|)
$$

We set, for any $\xi \in \Lambda_{x}$,

$$
\begin{aligned}
\mu & =|x z| \\
l & =l(\xi)=\left(1 / \mu+\frac{\mu}{6}\left(g_{x}-\epsilon\right)\right) \cdot\left(1 / \mu-\mu\left(g_{x}-\epsilon\right) / 3+H_{z}^{\delta} f(T \xi)\right)^{-1}
\end{aligned}
$$

and

$$
h_{x z}(\xi)=l^{2} \cdot H_{z}^{\delta} f(T \xi)+\frac{(l-1)^{2}}{\mu}-\frac{l^{2}+l+1}{3} \mu\left(g_{x}-\epsilon\right) .
$$

By noting (4.4) and that

$$
-\cot _{k_{0}}(|x \gamma(\ell)|) \leqslant-\cot _{k_{0}}(|z \gamma(\ell)|) \leqslant H_{z}^{\delta} f \leqslant \cot _{k_{0}}(|p z|) \leqslant \cot _{k_{0}}(|p x| / 2),
$$

we get $l(\xi)>0$ for $\mu$ is sufficiently small. Thus by Lemma 3.1, we have

$$
H_{x}^{\delta} f \leqslant h_{x z}
$$

Consequently,

$$
H_{x}^{\delta} f \leqslant h, \quad \text { on } \Lambda_{x}
$$


where $h=\min \left\{h_{x z}, \cot _{k_{0}}(|p x|)\right\}$. Then, by combining this with (4.1), we get

$$
\oint_{\Lambda_{x}} h \geqslant \cot _{K}(|p x|) .
$$

Therefore, by (4.5) and (4.7), there holds

$$
\begin{aligned}
\oint_{\Lambda_{x}} h-\oint_{\Lambda_{z}} H_{z}^{\delta} f & \geqslant \cot _{K}(|p x|)-\cot _{K}(|p z|) \\
& \geqslant-\mu\left(K+\cot _{K}^{2}(|p x|)\right)-C_{5} \mu^{2}
\end{aligned}
$$

where

$$
C_{5}=\max _{|p z| \leqslant t \leqslant|p x|}\left|\cot _{K}^{\prime \prime}(t)\right| \leqslant \max _{|p x| / 2 \leqslant t \leqslant|p x|}\left|\cot _{K}^{\prime \prime}(t)\right|
$$

On the other hand, rewriting Equation (4.6), we have

$$
\begin{aligned}
(1 / \mu & \left.-\mu\left(g_{x}-\epsilon\right) / 3+H_{z}^{\delta} f \circ T\right) \cdot h_{x z} \\
& =-\left(g_{x}-\epsilon\right)+H_{z}^{\delta} f \circ T \cdot\left(1 / \mu-\mu\left(g_{x}-\epsilon\right) / 3\right)+\left(\mu\left(g_{x}-\epsilon\right)\right)^{2} / 12 .
\end{aligned}
$$

By the facts that $h \leqslant h_{x z}$ and $1 / \mu-\mu\left(g_{x}-\epsilon\right) / 3+H_{z}^{\delta} f \circ T>0$, we get

$$
\begin{aligned}
(1 / \mu & \left.-\mu\left(g_{x}-\epsilon\right) / 3+H_{z}^{\delta} f \circ T\right) \cdot h \\
& \leqslant-\left(g_{x}-\epsilon\right)+H_{z}^{\delta} f \circ T \cdot\left(1 / \mu-\mu\left(g_{x}-\epsilon\right) / 3\right)+\left(\mu\left(g_{x}-\epsilon\right)\right)^{2} / 12 .
\end{aligned}
$$

That is,

$$
(1 / \mu-D) \cdot\left(h-H_{z}^{\delta} f \circ T\right) \leqslant-\left(g_{x}-\epsilon\right)-h^{2}+\left(\mu\left(g_{x}-\epsilon\right)\right)^{2} / 12,
$$

where $D=\mu\left(g_{x}-\epsilon\right) / 3-h$.

Denote that $C_{6}=\max |D|=\max \left|h+\mu\left(g_{x}-\epsilon\right) / 3\right|$, which is independent of $\mu$. Thus we get

$$
\begin{aligned}
\oint_{\Lambda_{x}} \frac{\epsilon-g_{x}}{1 / u-D} & =\oint_{\Lambda_{x}} \frac{\left(\epsilon-g_{x}\right)^{+}}{1 / u-D}-\oint_{\Lambda_{x}} \frac{\left(\epsilon-g_{x}\right)^{-}}{1 / u-D} \\
& \leqslant \frac{\oint_{\Lambda_{x}}\left(\epsilon-g_{x}\right)^{+}}{1 / u-C_{6}}-\frac{\oint_{\Lambda_{x}}\left(\epsilon-g_{x}\right)^{-}}{1 / u+C_{6}} \\
& =\frac{1 / \mu \oint_{\Lambda_{x}}\left(\epsilon-g_{x}\right)+C_{6} \oint_{\Lambda_{x}}\left|g_{x}-\epsilon\right|}{1 / \mu^{2}-C_{6}^{2}}
\end{aligned}
$$


By (4.4), (4.10) and the Ricci curvature condition that $\oint_{\Lambda_{x}} g_{x} \geqslant K-\epsilon$, we have

$$
\oint_{\Lambda_{x}} \frac{\epsilon-g_{x}}{1 / u-D} \leqslant \mu(2 \epsilon-K)+C_{7} \mu^{2}
$$

here and in the following of this proof, all of constants $C_{7}, C_{8}, C_{9}$ and $C_{10}$ are independent of $\mu$.

From (4.9) and (4.4), we get

$$
\oint_{\Lambda_{x}} h-\oint_{\Lambda_{x}} H_{z}^{\delta} f \circ T \leqslant \mu(2 \epsilon-K)+C_{7} \mu^{2}-\frac{\oint_{\Lambda_{x}} h^{2}}{1 / \mu+C_{6}}+\frac{\left(C_{4}+\epsilon\right)^{2} \mu^{2}}{1 / \mu-C_{6}} .
$$

By combining (4.8), (4.12) and noting that $T$ is an isometry, we have

$$
\oint_{\Lambda_{x}} h^{2} \leqslant \cot _{K}^{2}(|p x|)+2 \epsilon+C_{8} \mu
$$

Therefore,

$$
\oint_{\Lambda_{x}} h^{2} \leqslant \cot _{K}^{2}(|p x|)+3 \epsilon
$$

as $\mu$ suffices small.

Note that (4.12) implies

$$
\oint_{\Lambda_{x}} h \leqslant \oint_{\Lambda_{x}} H_{z}^{\delta} f \circ T+C_{9} \mu
$$

Using (4.5) and noting that $T$ is an isometry, we have

$$
\oint_{\Lambda_{x}} h \leqslant \cot _{K}(|p z|)+C_{9} \mu \leqslant \cot _{K}(|p x|)+\mu\left(K+\cot _{K}^{2}(|p z|)\right)+C_{9} \mu .
$$

Since $|p x| / 2<|p z|<|p x|$, we have

$$
\oint_{\Lambda_{x}} h \leqslant \cot _{K}(|p x|)+C_{10} \mu,
$$

Thus, when $\mu$ is sufficiently small, we get

$$
\oint_{\Lambda_{x}} h \leqslant \cot _{K}(|p x|)+\epsilon .
$$


By combining (4.7) and (4.14), we obtain

$$
\cot _{K}(|p x|) \cdot \oint_{\Lambda_{x}} h \geqslant \cot _{K}^{2}(|p x|)-\epsilon \cdot\left|\cot _{K}(|p x|)\right| .
$$

Hence, by (4.13) and (4.15), we have

$$
\oint_{\Lambda_{x}}\left(h-\cot _{K}(|p x|)\right)^{2} \leqslant\left(3+2\left|\cot _{K}(|p x|)\right|\right) \cdot \epsilon .
$$

This completes the proof of the claim.

Now let us continue the proof of the lemma.

Given any $\epsilon_{1}>0$, the above claim implies that the measure

$$
\begin{aligned}
& \nu\left(\left\{\xi \in \Lambda_{x}: H_{x}^{\delta} f \geqslant \cot _{K}+\epsilon_{1}\right\}\right) \\
& \quad \leqslant \nu\left(\left\{\xi \in \Lambda_{x}:\left|h-\cot _{K}(|p x|)\right| \geqslant \epsilon_{1}\right\}\right) \leqslant\left(3+2\left|\cot _{K}(|p x|)\right|\right) \epsilon / \epsilon_{1}^{2} .
\end{aligned}
$$

Letting $\epsilon \rightarrow 0^{+}$, by a standard diagonal argument, we can obtain a subsequence of $\delta$, still denoted by $\delta$, such that

$$
\nu\left(\left\{\xi \in \Lambda_{x}: H_{x}^{\delta} f \geqslant \cot _{K}+\epsilon_{1}\right\}\right)=0 .
$$

By the arbitrariness of $\epsilon_{1}$, after a further diagonal argument, we obtain a subsequence of $\delta$, denoted by $\delta$ again, such that

$$
\nu\left(\left\{\xi \in \Lambda_{x}: H_{x}^{\delta} f>\cot _{K}\right\}\right)=0 .
$$

Thus we have

$$
H_{x}^{\delta} f \leqslant \cot _{K}(|p x|)
$$

almost everywhere in $\Lambda_{x}$.

Finally, by combining (4.1) and the definition of $\Delta^{\delta} f$, we conclude that

$$
H_{x}^{\delta} f=\cot _{K}(|p x|)
$$

almost everywhere in $\Lambda_{x}$. Therefore we have completed the proof of the lemma.

In order to deal with the zero-measure set in the above lemma, we need the following segment inequality of Cheeger and Colding [6]. See also [35] for a statement that is stronger than the following proposition. 
Proposition 4.1 (Segment inequality). Let $M$ be an $n$-dimensional Alexandrov space with curvature $\geqslant k_{0}$ (for some constant $k_{0}<0$ ). Let $A_{1}$, $A_{2} \subset M$ be two open sets, and let $\gamma_{y_{1}, y_{2}}$ be a geodesic from $y_{1}$ to $y_{2}$ with arc-parametrization. Assume $W \subset M$ is an open set with

$$
\bigcup_{y_{1} \in A_{1}, y_{2} \in A_{2}} \gamma_{y_{1}, y_{2}} \subset W
$$

If e be a non-negative integrable function on $W$, then

$$
\int_{A_{1} \times A_{2}} \int_{0}^{\left|y_{1} y_{2}\right|} e\left(\gamma_{y_{1}, y_{2}}(s)\right) d s \leqslant C\left(n, k_{0}, D\right) \cdot D \cdot\left(\operatorname{vol}\left(A_{1}\right)+\operatorname{vol}\left(A_{2}\right)\right) \int_{W} e,
$$

where $D=\sup _{y_{1} \in A_{1}, y_{2} \in A_{2}}\left|y_{1} y_{2}\right|$ and

$$
C\left(n, k_{0}, D\right)=\left(\sinh \left(\sqrt{-k_{0}} D\right) / \sinh \left(\sqrt{-k_{0}} D / 2\right)\right)^{n-1} .
$$

We now define the upper Hessian of $f, \overline{\operatorname{Hess}}_{x} f: T_{x} \rightarrow \mathbb{R} \cup\{-\infty\}$ by

$$
\overline{\operatorname{Hess}}_{x} f(v, v) \stackrel{\text { def }}{=} \limsup _{s \rightarrow 0} \frac{f \circ \exp _{x}(s \cdot v)-f(x)-d_{x} f(v) \cdot s}{s^{2} / 2}
$$

for any $v \in T_{x}$.

Clearly, this definition also works for any semi-concave function on $M$. If $u$ is a $\lambda$-concave function, then its upper Hessian $\overline{\operatorname{Hess}}_{x} u(\xi, \xi) \leqslant \lambda$ for any $\xi \in \Sigma_{x}$.

For a semi-concave function $u$, we denote its regular set $\operatorname{Reg}_{u}$ by

$$
\operatorname{Reg}_{u}:=\{x \in M: \text { there exists Perelman's Hessian of } u \text { at } x\} .
$$

It was showed in [29] that $\operatorname{Reg}_{u}$ has full measure for any semi-concave function $u$. It is clear that $\overline{\operatorname{Hess}}_{x} u=\operatorname{Hess}_{x} u$ for any $x \in \operatorname{Reg}_{u}$.

Definition 4.1. Let $p \in M$. The cut locus of $p$, denoted by $\mathrm{Cut}_{p}$, is defined to be the set all of points $x$ in $M$ such that geodesic $p x$, from $p$ to $x$, can not be extended. $[26])$.

It was shown in [24] that $\mathrm{Cut}_{p}$ has zero (Hausdorff) measure (see also

Set $W_{p}=M \backslash\left(\{p\} \cup \mathrm{Cut}_{p}\right)$. For any two points $x, y \in M$ with $x \neq y$, a direction from $x$ to $y$ is denoted by $\uparrow_{x}^{y}$. 
The following two lemmas are concerned with the rigidity part of Theorem 3.1 .

Lemma 4.2. Let $M$ be an $n$-dimensional Alexandrov space with Ricci curvature $\geqslant(n-1) K$ and let $f=$ dist $_{p}$. Suppose that $B_{p}(R) \backslash\{p\} \subset W_{p}$ for some $0<R \leqslant \pi / \sqrt{K}$ (if $K \leqslant 0$, we set $\pi / \sqrt{K}$ to be $+\infty$ ). Assume that for each $x \in B_{p}(R) \backslash\{p\}$, there exists a sequence $\theta:=\left\{\theta_{j}\right\}_{j=1}^{\infty} \in \mathcal{S}$ such that

$$
\Delta^{\theta^{\prime}} f(x)=(n-1) \cdot \cot _{K}(|p x|)
$$

for any subsequence $\theta^{\prime} \subset \theta$.

Then the function $\varrho_{K} \circ f$ is $\left(1-K \cdot \varrho_{K} \circ f\right)$-concave in $B_{p}(R) \backslash\{p\}$.

Proof. It suffices to show one variable function $h_{p}:=\varrho_{K} \circ f \circ \gamma(s)$ satisfies that

$$
h_{p}^{\prime \prime} \leqslant 1-K h_{p}
$$

for any geodesic $\gamma(s) \subset B_{p}(R) \backslash\{p\}$. Let $\chi(s)$ be an continuous function on an open interval $(a, b)$. Here and in the sequel we write $\chi^{\prime \prime}(s) \leqslant B$ for $s \in(a, b)$ if $\chi(s+\tau) \leqslant \chi(s)+A \cdot \tau+B \cdot \tau^{2} / 2+o\left(\tau^{2}\right)$ for some $A \in \mathbb{R} . \chi^{\prime \prime}(s)<+\infty$ means that $\chi^{\prime \prime}(s) \leqslant B$ for some $B \in \mathbb{R}$. If $\chi_{1}$ is another continuous function on $(a, b)$, then $\chi^{\prime \prime} \leqslant \chi_{1}$ means $\chi^{\prime \prime}(s) \leqslant \chi_{1}(s)$ for all $s \in(a, b)$.

Fix a geodesic $\gamma \subset B_{p}(R) \backslash\{p\}$. Let $x=\gamma(0), y=\gamma(l)$. Without loss of generality, we can assume that $\gamma$ is the unique geodesic from $x$ to $y$ and

$$
|p x|+|p y|+|x y|<2 R \text {. }
$$

We consider the function $u: W_{p} \rightarrow \mathbb{R}^{+} \cup\{0\}$,

$$
u(z)=\sup _{\xi \in \Sigma_{z}}\left|\overline{\operatorname{Hess}}_{z} f(\xi, \xi)-\cot _{K}(|p z|) \cdot \sin ^{2}\left(\left|\xi, \uparrow_{z}^{p}\right|\right)\right|
$$

For any point $z \in \operatorname{Reg}_{f} \cap B_{p}(R), \overline{\operatorname{Hess}}_{z} f$ is a bilinear form on $T_{z}$ and $\overline{\operatorname{Hess}}_{z} f\left(\uparrow_{z}^{p}, \uparrow_{z}^{p}\right)=0$. Let

$$
\Lambda_{z}=\left\{\xi \in \Sigma_{z}: \angle\left(\xi, \uparrow_{z}^{p}\right)=\pi / 2\right\}
$$

By Lemma 4.1, we have $\overline{\operatorname{Hess}}_{z} f(\xi, \xi)=H_{z}^{\delta} f=\cot _{K}(|p z|)$ on $\Lambda_{z}$ for some subsequence $\delta$ of $\theta$, and hence $u(z)=0$.

Since $\operatorname{Reg}_{f}$ has full measure in $B_{p}(R)$, we conclude that $u \equiv 0$ almost everywhere in $B_{p}(R)$. 
Given any positive number $\epsilon>0$ such that

$$
\epsilon \ll \min \{|p x|,|p y|,|x y|, 2 R-(|p x|+|p y|+|x y|)\} .
$$

Let $x_{1} \in B_{x}(\epsilon)$ and $y_{1} \in B_{y}(\epsilon)$, and let $\gamma_{x_{1}, y_{1}}(s)$ be a geodesic from $x_{1}$ to $y_{1}$. By triangle inequality, it is easy to see

$$
\left|p x_{1}\right|+\left|x_{1} y_{1}\right|+\left|p y_{1}\right|<2 R
$$

as $\epsilon$ is sufficiently small. Thus $\gamma_{x_{1}, y_{1}} \in B_{p}(R)$.

Set $u_{x_{1}, y_{1}}(s)=u\left(\gamma_{x_{1}, y_{1}}(s)\right)$. By applying Proposition 4.1 to $A_{1}=B_{x}(\epsilon)$, $A_{2}=B_{y}(\epsilon), W=B_{p}(R)$ and function $u$, we know that there exist two points $x_{1} \in B_{x}(\epsilon)$ and $y_{1} \in B_{y}(\epsilon)$ such that $u_{x_{1}, y_{1}}(s)=0$ almost everywhere on $\left(0,\left|x_{1} y_{1}\right|\right)$.

Consider a $s_{0} \in\left(0,\left|x_{1}, y_{1}\right|\right)$ such that $u_{x_{1}, y_{1}}\left(s_{0}\right)=0$. Set $z=\gamma_{x_{1}, y_{1}}\left(s_{0}\right)$, $\zeta^{+}=\gamma_{x_{1}, y_{1}}^{+}\left(s_{0}\right)$ and $\zeta^{-}=\gamma_{x_{1}, y_{1}}^{-}\left(s_{0}\right)$. Then we have

$$
\overline{\operatorname{Hess}}_{z} f\left(\zeta^{+}, \zeta^{+}\right)=\overline{\operatorname{Hess}}_{z} f\left(\zeta^{-}, \zeta^{-}\right)=\cot _{K}(|p z|) \sin ^{2}\left(\left|\zeta^{+}, \uparrow_{z}^{p}\right|\right)
$$

Therefore, for function $\widetilde{f}(s)=f \circ \gamma_{x_{1}, y_{1}}(s)$, we get

$$
\begin{aligned}
\tilde{f}\left(h+s_{0}\right) & \leqslant \tilde{f}\left(s_{0}\right)+h \tilde{f}^{+}\left(s_{0}\right)+F\left(s_{0}\right) \cdot h^{2} / 2+o\left(h^{2}\right), \\
\tilde{f}\left(-h+s_{0}\right) & \leqslant \widetilde{f}\left(s_{0}\right)-h \tilde{f}^{-}\left(s_{0}\right)+F\left(s_{0}\right) \cdot h^{2} / 2+o\left(h^{2}\right),
\end{aligned}
$$

for any $h>0$, where

$$
F\left(s_{0}\right)=\cot _{K}(|p z|) \cdot \sin ^{2}\left(\left|\zeta^{+}, \uparrow_{z}^{p}\right|\right)=\cot _{K}(|p z|) \cdot\left(1-\cos ^{2}\left(\left|\zeta^{+}, \uparrow_{z}^{p}\right|\right)\right) .
$$

By the first variation formula of arc-length, we have

$$
\tilde{f}^{+}(s)=-\cos \left(\left|\zeta^{+}, \uparrow_{z}^{p}\right|\right) \text { and } \tilde{f}^{-}(s)=-\cos \left(\left|\zeta^{-}, \uparrow_{z}^{p}\right|\right)
$$

Note that $\gamma_{x_{1}, y_{1}} \in W_{p}$,

$$
\left|\zeta^{+}, \uparrow_{z}^{p}\right|+\left|\zeta^{-}, \uparrow_{z}^{p}\right|=\pi
$$

which implies that $\widetilde{f}(s)$ is continuously differential. Then by combining this with (4.19), we have

$$
\widetilde{f}^{\prime \prime}(s) \leqslant F(s)=\cot _{K} \widetilde{f}(s) \cdot\left(1-\widetilde{f}^{2}(s)\right)
$$


for almost everywhere $s \in\left(0,\left|x_{1} y_{1}\right|\right)$. Thus the function $\widetilde{h}(s)=\varrho_{K} \circ \tilde{f}(s)$ satisfies

$$
\widetilde{h}^{\prime \prime}(s) \leqslant 1-K \widetilde{h}(s)
$$

for almost everywhere $s \in\left(0,\left|x_{1} y_{1}\right|\right)$. On the other hand, the fact $f$ is semiconcave implies that $\tilde{h}^{\prime \prime}(s)<+\infty$ for all $s \in\left(0,\left|x_{1} y_{1}\right|\right)$. Thus, from $1.3(3)$ in [34], we have

$$
\widetilde{h}^{\prime \prime} \leqslant 1-K \widetilde{h}
$$

Letting $\epsilon \rightarrow 0^{+}$, we can get point sequences $\left\{x_{i}\right\}$ and $\left\{y_{i}\right\}$ such that $x_{i} \rightarrow x, y_{i} \rightarrow y$ and

$$
\widetilde{h}_{i}^{\prime \prime} \leqslant 1-K \widetilde{h}_{i}
$$

where $\widetilde{h}_{i}=\varrho_{K} \circ f \circ \gamma_{x_{i}, y_{i}}(s)$. Since the geodesic from $x$ to $y$ is unique, there exists a subsequence of geodesics $\gamma_{x_{i}, y_{i}}$, which converges to geodesic $\gamma$ uniformly. Hence $\widetilde{h}_{i}$ converges to $h$ uniformly, and the desired result follows from 1.3(4) in [34]. Therefore, we have completed the proof.

Lemma 4.3. Let $\sigma(t)$ and $\varsigma(t)$ be two geodesics in $B_{p}(R)$ with $\sigma(0)=$ $\varsigma(0)=p$, and let

$$
\varphi\left(\tau, \tau^{\prime}\right)=\widetilde{\angle}_{K} \sigma(\tau) p \varsigma\left(\tau^{\prime}\right)
$$

be the comparison angle of $\angle \sigma(\tau) p \varsigma\left(\tau^{\prime}\right)$ in the $K$-plane. Then, under the same assumptions as Lemma 4.2, we have $\varphi\left(\tau, \tau^{\prime}\right)$ is non-increasing with respect to $\tau$ and $\tau^{\prime}$.

(If $K>0$, we add the assumption that $\left.\tau+\tau^{\prime}+\left|\sigma(\tau) \varsigma\left(\tau^{\prime}\right)\right|<2 \pi / \sqrt{K}\right)$.

Proof. Firstly, we claim that for any triangle $\triangle p x y$ (if $K>0$, we assume that $|p x|+|p y|+|x y|<2 \pi / \sqrt{K})$, there exists a comparison triangle $\triangle \bar{p} \bar{x} \bar{y}$ in the $K$-plane $M_{K}^{2}$ such that

$$
\angle \bar{p} \bar{x} \bar{y} \leqslant \angle p x y, \quad \angle \bar{p} \bar{y} \bar{x} \leqslant \angle p y x .
$$

Indeed, for any triangle $\triangle p x y \in B_{p}(R)$, there exists a triangle $\triangle \widehat{p} \widehat{x} \widehat{y}$ in $M_{K}^{2}$ such that

$$
|\widehat{p} \widehat{x}|=|p x|, \quad|\widehat{x} \widehat{y}|=|x y|, \quad \angle \widehat{p} \widehat{x} \widehat{y}=\angle p x y,
$$


and by Lemma 4.2 , we have

$$
|\widehat{p} \widehat{y}| \geqslant|p y|
$$

So by an obvious reason, we get the required triangle $\triangle \bar{p} \bar{x} \bar{y}$.

Fix $\tau^{\prime}>0$ and write $\varsigma=\varsigma\left(\tau^{\prime}\right)$. We only need to show $\varphi(\tau):=\varphi\left(\tau, \tau^{\prime}\right)$ is non-increasing with respect to $\tau$.

Let $\triangle \bar{\sigma}(\tau) \bar{p} \bar{\varsigma}$ be a comparison triangle of $\triangle \sigma(\tau) p \varsigma$ in the $K$-plane $M_{K}^{2}$ and extend the geodesic $\bar{p} \bar{\sigma}(\tau)$ slightly longer to $\bar{\sigma}(\tau+s)$ for small $s>0$.

Since the function dist $_{\varsigma}$ is $\lambda$-concave for some number $\lambda \in \mathbb{R}$, we have

$$
|\varsigma \sigma(\tau+s)| \leqslant|\varsigma \sigma(\tau)|+s \cdot(-\cos \angle \sigma(\tau+s) \sigma(\tau) \varsigma)+s^{2} \lambda / 2
$$

On the other hand, we have

$$
|\bar{\zeta} \bar{\sigma}(\tau+s)|=|\bar{\zeta} \bar{\sigma}(\tau)|+s \cdot(-\cos \angle \bar{\sigma}(\tau+s) \bar{\sigma}(\tau) \bar{\zeta})+s^{2} \bar{\lambda} / 2+o\left(s^{2}\right)
$$

for some number $\bar{\lambda} \in \mathbb{R}$. Note from (4.20) that

$$
\angle \bar{\sigma}(\tau+s) \bar{\sigma}(\tau) \bar{\varsigma} \geqslant \angle \sigma(\tau+s) \sigma(\tau) \varsigma
$$

By combining this with (4.21), (4.22) and $|\varsigma \sigma(\tau)|=|\bar{\varsigma} \bar{\sigma}(\tau)|$, we have

$$
|\varsigma \sigma(\tau+s)| \leqslant|\bar{\zeta} \bar{\sigma}(\tau+s)|+(-\lambda+\bar{\lambda}) s^{2}+o\left(s^{2}\right)
$$

Now, if $K>0$, by cosine law in $M_{K}^{2}$, we have

$$
\begin{aligned}
\cos \widetilde{\angle}_{K} \sigma(\tau+s) p \varsigma-\cos \widetilde{\angle}_{K} \sigma(\tau) p \varsigma \\
=\frac{\cos (\sqrt{K}|\varsigma \sigma(\tau+s)|)-\cos (\sqrt{K}|\bar{\zeta} \bar{\sigma}(\tau+s)|)}{\sin (\sqrt{K}|p \sigma(\tau+s)|) \cdot \sin (\sqrt{K}|p \varsigma|)} \\
\geqslant \frac{-(\lambda+\bar{\lambda})}{\sin (\sqrt{K}|p \sigma(\tau+s)|) \cdot \sin (\sqrt{K}|p \varsigma|)} \cdot s^{2} .
\end{aligned}
$$

Hence, we get

$$
\frac{d^{+}}{d \tau} \cos \widetilde{\angle}_{K} \sigma(\tau) p \varsigma \geqslant 0
$$

If $K \leqslant 0$, using a similar argument, we can get $\frac{d^{+}}{d \tau} \widetilde{L}_{K} \sigma(\tau) p \varsigma \leqslant 0$. Therefore we have completed the proof of the lemma. 


\section{Maximal diameter theorem}

The main purpose of this section is to prove Theorem 1.4.

Bonnet-Myers' theorem asserts that if an $n$-dimensional Riemannian manifold has Ric $\geqslant n-1$, then its diameter $\leqslant \pi$. Furthermore, its fundamental group is finite.

The first assertion, the diameter estimate, has been extend to metric measure space with $\operatorname{CD}(n, n-1)$ (see [38]) or $\operatorname{MCP}(n, n-1)$ (see [20]). Since our condition Ric $\geqslant n-1$ implies the curvature-dimension condition $\mathrm{CD}(n, n-1)$, the first assertion of Bonnet-Myers' theorem also holds on an $n$-dimensional Alexandrov space $M$ with $\operatorname{Ric}(M) \geqslant n-1$ and $\partial M=\varnothing$.

Now we consider the second assertion: finiteness of the fundamental group.

Proposition 5.1. Let $M$ be an $n$-dimensional Alexandrov space without boundary and $\operatorname{Ric}(M) \geqslant n-1$. The order of fundamental group of $M$, $\operatorname{ord} \pi_{1}(M)$, satisfies

$$
\operatorname{ord} \pi_{1}(M) \leqslant \frac{\omega_{n}}{\operatorname{vol}(M)}
$$

where $\omega_{n}$ is the volume of $n$-dimensional standard sphere $\mathbb{S}^{n}$. In particular, if add assumption $\operatorname{vol}(M)>\omega_{n} / 2, M$ is simply connected.

Proof. Let $\widetilde{M}$ be the universal covering of $M$. We have $\operatorname{Ric}(\widetilde{M}) \geqslant n-1$. Therefore, by Bishop-Gromov volume comparison theorem (see Corollary A.1 in the Appendix), we get

$$
\operatorname{ord} \pi_{1}(M) \cdot \operatorname{vol}(M)=\operatorname{vol}(\widetilde{M}) \leqslant \omega_{n}
$$

This completes the proof.

Now, we are in position to prove Theorem 1.4. We rewrite it as following

Theorem 5.1. Let $M$ be an $n$-dimensional Alexandrov space with $\operatorname{Ric}(M) \geqslant$ $n-1$ and $\partial M=\varnothing$. If $\operatorname{diam}(M)=\pi$, then $M$ is isometric to suspension $[0, \pi] \times{ }_{\sin } N$, where $N$ is an Alexandrov space with curvature $\geqslant 1$.

Proof. Takes two points $p, q \in M$ such that $|p q|=\pi$.

Exactly as in Riemannian manifold case, by using Bishop-Gromov volume comparison theorem, we have the following assertions: 
Fact: (i) For any point $x \in M$, there holds $|p x|+|q x|=\pi$. This implies $W_{p}=W_{q}=M \backslash\{p, q\}$.

(ii) For any $x \in M$, we can extend the geodesic $p x$ to a geodesic from $p$ to $q$. We will denote it by $p x q$.

(iii) For any non-degenerate triangle $\triangle p x y$, we have $|p x|+|p y|+|x y|<$ $2 \pi$.

(iv) For any direction $\xi \in \Sigma_{p}$, there exists a geodesic $\gamma_{\xi}$ such that $\gamma_{\xi}(0)=$ $p, \gamma_{\xi}^{+}(0)=\xi$ and its length is equal to $\pi$.

Indeed, the first assertion (i) is an immediate consequence of BishopGromov volume comparison theorem (see, for example,[27, p. 271]). Gluing geodesics $p x$ and $q x$, the result curve has Length $=\pi=|p q|$. Thus it is a geodesic. This proves the second assertion (ii). The third assertion (iii) follows directly from triangle inequality

$$
|p x|+|p y|+|x y|<|p x|+|p y|+|q x|+|q y| \leqslant 2 \pi .
$$

To show (iv), we consider a sequence of direction $\xi_{i} \in \Sigma_{p}$ such that $\xi_{i} \rightarrow \xi$ and there exists geodesics $\alpha_{i}$ with $\alpha_{i}(0)=p$ and $\alpha_{i}^{+}(0)=\xi_{i}$. From (ii), we can extend each $\alpha_{i}$ to a new geodesic with Length $=\pi$, denoted by $\alpha_{i}$ again. By Arzela-Ascoli Theorem, we can take a limit from some subsequence of $\alpha_{i}$. Clearly, the limit is the desired geodesic. This proves the last assertion (iv).

Let $f=\operatorname{dist}_{p}$ and $\bar{f}=\operatorname{dist}_{q}$. For any point $x \neq p, q$, we set $\Lambda_{x} \subset \Sigma_{x}$ all of directions which are vertical with the geodesic $p x q$.

Fix a sequence $\theta=\left\{\theta_{j}\right\}_{j=1}^{\infty} \in \mathcal{S}$. By Theorem 3.1, we can find a subsequence $\delta \subset \theta$ such that

$$
\Delta^{\delta} f(x) \leqslant(n-1) \cdot \cot (|p x|) \quad \text { and } \quad \Delta^{\delta} \bar{f}(x) \leqslant(n-1) \cdot \cot (|q x|) .
$$

The above fact (i) implies $f+\bar{f}=\pi$. Thus

$$
H_{x}^{\delta} \bar{f}(\xi)=-\liminf _{s \rightarrow 0} \frac{f \circ \exp _{x}(s \cdot \xi)-f(x)}{s^{2} / 2} .
$$

By Definition 3.1, we have $H_{x}^{\delta^{\prime}} f \geqslant-H_{x}^{\delta} \bar{f}$ for any subsequence $\delta^{\prime} \subset \delta$. Hence, by combining this with (5.1) and the definition of $\Delta^{\delta} f$, we get

$$
\Delta^{\delta^{\prime}} f(x) \geqslant-\Delta^{\delta} \bar{f}(x) \geqslant-(n-1) \cot (|q x|)=(n-1) \cdot \cot (|p x|) .
$$

Note also that

$$
\Delta^{\delta^{\prime}} f(x) \leqslant \Delta^{\delta} f(x)
$$


By combining this with (5.1), this implies that

$$
\Delta^{\delta^{\prime}} f(x)=(n-1) \cot (|p x|)
$$

for any subsequence $\delta^{\prime} \subset \delta$.

From Lemma $4.2,-\cos f$ is $\cos f$-concave in $B_{p}(\pi) \backslash\{p\}=W_{p}$. Given any geodesic $\sigma(s):[0, L] \rightarrow W_{p}$ with $L<\pi$, we have

$$
(-\cos f \circ \sigma)^{\prime \prime}(s) \leqslant \cos f \circ \sigma(s), \quad \forall s \in(0, L) .
$$

Similarly, $-\cos \bar{f}$ is $\cos \bar{f}$-concave in $W_{q}=W_{p}$ and

$$
(-\cos \bar{f} \circ \sigma)^{\prime \prime}(s) \leqslant \cos \bar{f} \circ \sigma(s), \quad \forall s \in(0, L) .
$$

Since $f+\bar{f}=\pi, \cos f=-\cos \bar{f}$, by combining this with (5.3) and (5.4), we get

$$
(-\cos f \circ \sigma)^{\prime \prime}(s)=\cos f \circ \sigma(s), \quad \forall s \in(0, L) .
$$

Denote by

$$
M^{+}=\{x \in M: f(x) \leqslant \pi / 2\}, \quad M^{-}=\{x \in M: f(x) \geqslant \pi / 2\}
$$

and $N=M^{+} \cap M^{-}=\{x \in M: f(x)=\pi / 2\}$. Set

$$
v_{x}=(\text { geodesic } p x q) \cap N
$$

which is consisting of a single point.

We claim that $N$ is totally geodesic in $M$.

Indeed, take any two points $v_{1}, v_{2} \in N$ with $\left|v_{1} v_{2}\right|<\pi$. Let $\sigma(s)$ be a geodesic connected $v_{1}$ and $v_{2}$. By (5.5) and noting that

$$
\cos f\left(v_{1}\right)=\cos f\left(v_{2}\right)=0
$$

we have $\cos f \circ \sigma(s) \equiv 0$. This tells us $\sigma \subset N$ and $N$ is totally geodesic.

Now we are ready to prove that $M$ is isometric to suspension $[0, \pi] \times{ }_{\sin } N$. Consider any two points $x, y \in M \backslash\{p, q\}$. 
If $x, y \in M^{+}$, we know from Lemma 4.3 that

$$
\widetilde{\angle}_{1} x p y \geqslant \widetilde{L}_{1} v_{x} p v_{y} \text { and } \widetilde{\leftarrow}_{1} x q y \leqslant \widetilde{L}_{1} v_{x} q v_{y} .
$$

Note from Fact (i) that

$$
\widetilde{\angle}_{1} x p y=\widetilde{\angle}_{1} x q y .
$$

Thus we obtain

$$
\widetilde{\leftarrow}_{1} x p y=\widetilde{\leftarrow}_{1} v_{x} p v_{y}
$$

Clearly, if $x, y \in M^{-}$, the same argument also deduces the equality (5.7). While if $x \in M^{+}$and $y \in M^{-}$, by Lemma 4.3 again, we have

$$
\widetilde{L}_{1} x p y \geqslant \widetilde{L}_{1} v_{x} p y=\widetilde{L}_{1} v_{x} p v_{y} \text { and } \widetilde{L}_{1} x p y \leqslant \widetilde{L}_{1} x p v_{y}=\widetilde{L}_{1} v_{x} p v_{y},
$$

which implies the equality (5.7).

Then by applying the cosine law to the comparison triangle, we get

$$
\cos (|x y|)=\cos (|p x|) \cdot \cos (|p y|)+\sin (|p x|) \cdot \sin (|p y|) \cos \widetilde{\measuredangle}_{1} v_{x} p v_{y} .
$$

This proves that $M$ is isometric to suspension $[0, \pi] \times \sin N$.

It remains to show that $N$ has curvature $\geqslant 1$.

We define a map $\Phi: N \rightarrow \Sigma_{p}$ by

$$
\Phi(v)=\uparrow_{p}^{v}, \quad \forall v \in N
$$

Since $N \subset W_{p}$ and $|p v|=\pi / 2$ for all $v \in N, \Phi$ is well defined.

Given two points $v_{1}, v_{2} \in N$, for any $x_{1} \in M$ lies in geodesic $p v_{1} q$ and any $x_{2} \in M$ lies in geodesic $p v_{2} q$, the equality (5.7) implies

$$
\widetilde{\angle}_{1} x_{1} p y_{1}=\widetilde{\angle}_{1} v_{1} p v_{2}=\left|v_{1} v_{2}\right|
$$

Since $\angle v_{1} p v_{2}=\lim _{x_{1} \rightarrow p, x_{2} \rightarrow p} \widetilde{L}_{1} x_{1} p y_{1}$, we have

$$
\left|\uparrow_{p}^{v_{1}} \uparrow_{p}^{v_{2}}\right|_{\Sigma_{p}}=\left|v_{1} v_{2}\right|
$$

This shows that $\Phi$ is an isometrical embedding. On the other hand, by Fact (iv), $\Phi$ is surjective. Therefore, $\Phi$ is an isometry. Thus $N$ has curvature $\geqslant 1$. Therefore, we have completed the proof of the theorem. 
Corollary 5.1. Let $M$ be an $n$-dimensional Alexandrov space with $\operatorname{Ric}(M)$ $\geqslant n-1$ and $\partial M=\varnothing$. If $\operatorname{rad}(M)=\pi$, then $M$ is isometric to the sphere $\mathbb{S}^{n}$ with standard metric.

Proof. For any point $p \in M$, there exists a point $q$ such that $|p q|=\pi$. From the proof of Theorem 5.1, we have that $-\cos _{\text {dist }} p$ is $\cos _{\text {dist }}$-concave in $B_{p}(\pi) \backslash\{p\}$. Thus $M$ has curvature $\geqslant 1$. It is well known (see, for example, [3, Lemma 10.9.10]) that an $n$-dimensional Alexandrov space with curvature $\geqslant 1$ and $\mathrm{rad}=\pi$ must be isometric to the sphere $\mathbb{S}^{n}$ with standard metric.

Remark 5.1. Colding in [5] had proved the corollary for limit spaces of Riemannian manifolds. That is, if $M_{i}$ is a sequence of $m$-dimensional Riemannian manifolds with $\operatorname{Ric}_{M_{i}} \geq m-1$ and converging to a metric space $X$ with $\operatorname{rad}_{X}=\pi$, then $X$ is isometric to the sphere $\mathbb{S}^{m^{\prime}}$ with standard metric for some integer $m^{\prime} \leqslant m$.

\section{Splitting theorem}

In this section, $M$ will always denote an $n$-dimensional Alexandrov space with curvature bounded below locally, $\operatorname{Ric}(M) \geqslant 0$ and $\partial M=\varnothing$. The main purpose of this section is to prove Theorem 1.3.

A curve $\gamma:[0,+\infty) \rightarrow M$ is called a ray if $|\gamma(s) \gamma(t)|=s-t$ for any $0 \leqslant$ $t<s<+\infty$. A curve $\gamma:(-\infty,+\infty) \rightarrow M$ is called a line if $|\gamma(s) \gamma(t)|=s-$ $t$ for any $-\infty<t<s<+\infty$. For a line $\gamma$, obviously, $\left.\gamma\right|_{[0,+\infty)}$ and $\left.\gamma\right|_{(-\infty, 0]}$ form two rays.

Given a ray $\gamma(t)$, we define the Busemann function $b_{\gamma}$ for $\gamma$ on $M$ by

$$
b_{\gamma}(x)=\lim _{t \rightarrow+\infty}(t-|x \gamma(t)|) .
$$

Clearly, it is well defined and is a 1-Lipschitz function.

From now on, in this section, we fix a line $\gamma(t)$ in $M$ and set $\gamma_{+}=$ $\left.\gamma\right|_{[0,+\infty)}, \gamma_{-}=\left.\gamma\right|_{(-\infty, 0]}$. Let $b_{+}$and $b_{-}$be the Busemann functions for rays $\gamma_{+}$and $\gamma_{-}$, respectively.

Let us recall what is the proof of the splitting theorem in the smooth case. When $M$ is a smooth Riemannian manifold, Cheeger-Gromoll in [9] used the standard Laplacian comparison and the maximum principle to conclude that $b_{+}$and $b_{-}$are harmonic on $M$. Then the elliptic regularity theory implies that they are smooth. The important step is to use Bochner formula to show that both $\nabla b_{+}$and $\nabla b_{-}$are parallel. Consequently, the 
splitting theorem follows directly from de Rham decomposition theorem. In [10], Eschenburg-Heintze gave a proof avoiding the elliptic regularity, while the Bochner formula is essentially used. But for the general Alexandrov spaces case, the main difficulty is the lack of Bochner formula.

We begin with a lemma which was proved by Kuwae and Shioya for Alexandrov spaces with $\operatorname{MCP}(n, 0)$ and hence for Alexandrov spaces with non-negative Ricci curvature. (See Lemma 6.5 and the proof of Theorem 1.3 in [12]).

Lemma 6.1. $b_{+}(x)+b_{-}(x) \equiv 0$, on $M$.

Lemma 6.2. For any point $x \in M$, there exists a unique line $\gamma_{x}$ such that $x=\gamma_{x}(0)$ and $b_{+} \circ \gamma_{x}$ is a linear function with $\left(b_{+} \circ \gamma_{x}\right)^{\prime}=1$.

Proof. Existence. If $x \in \gamma$, then we can write $x=\gamma\left(t_{0}\right)$. Hence we set $\gamma_{x}(t)=$ $\gamma\left(t+t_{0}\right)$, which is a desired line.

We then consider the case $x \notin \gamma$. Let $\sigma_{t,+}(s)$ be a geodesic from $x$ to $\gamma_{+}(t)$. By using Arzela-Ascoli Theorem, we can take a sequence $t_{j} \rightarrow+\infty$ such that $\sigma_{t_{j},+}$ converges to a limit curve $\sigma_{\infty,+}(s):[0,+\infty) \rightarrow M$. It is easy to check (see, for example,[27, p. 286]) that $\sigma_{\infty,+}$ is 1-Lipschitz and

$$
b_{+} \circ \sigma_{\infty,+}(s)=s+b_{+} \circ \sigma_{\infty,+}(0)=s+b_{+}(x) \text { for all } s \geqslant 0 .
$$

By a similar construction, we can obtain a 1-Lipschitz curve $\sigma_{\infty,-}\left(s^{\prime}\right)$ : $(-\infty, 0] \rightarrow M$ such that $\sigma_{\infty,-}(0)=x$ and

$$
b_{-} \circ \sigma_{\infty,-}\left(s^{\prime}\right)=-s^{\prime}+b_{-}(x) \text { for all } s^{\prime} \leqslant 0 .
$$

Let $\sigma_{\infty}=\sigma_{\infty,+} \cup \sigma_{\infty,-}:(-\infty,+\infty) \rightarrow M$. This is a 1-Lipschitz curve. By Lemma 6.1, we have

$$
b_{+} \circ \sigma_{\infty}(s)=s+b_{+}(x) \text { for all } s \in(-\infty,+\infty) .
$$

Then for any $-\infty<t<s<\infty$, by (6.1), we get

$$
s-t=b_{+} \circ \sigma_{\infty}(s)-b_{+} \circ \sigma_{\infty}(t) \leqslant\left|\sigma_{\infty}(s) \sigma_{\infty}(t)\right| \leqslant s-t .
$$

Thus $\sigma_{\infty}$ is a line. Equation (6.1) shows that it is a desired line.

Uniqueness. Argue by contradiction. Suppose that there exist two such lines $\gamma_{1}, \gamma_{2}$. 
The equations $\left(b_{+} \circ \gamma_{1}\right)^{\prime}=\left(b_{+} \circ \gamma_{2}\right)^{\prime}=1$ implies

$$
b_{+} \circ \gamma_{1}(-1)=b_{+}(x)-1 \quad \text { and } \quad b_{+} \circ \gamma_{2}(1)=b_{+}(x)+1
$$

Hence

$$
b_{+} \circ \gamma_{2}(1)-b_{+} \circ \gamma_{1}(-1)=2
$$

Since $b_{+}$is 1-Lipschitz, we get

$$
\left|\gamma_{1}(-1) \gamma_{2}(1)\right| \geqslant b_{+} \circ \gamma_{2}(1)-b_{+} \circ \gamma_{1}(-1)=2
$$

On the other hand,

$$
\operatorname{Length}\left(\gamma_{1}([-1,0]) \cup \gamma_{2}([0,1])\right)=2 \text {. }
$$

Thus $\gamma_{1}([-1,0]) \cup \gamma_{2}([0,1])$ is a geodesic. This contradicts to that $M$ is nonbranching. The proof of the lemma is completed.

For any point $x \in M$, we take the line $\gamma_{x}$ in Lemma 6.2. Let

$$
\begin{aligned}
& L_{x}=\left\{\xi \in T_{x} \mid \angle\left(\xi, \gamma_{x}^{+}(0)\right)=\angle\left(\xi, \gamma_{x}^{-}(0)\right)=\pi / 2\right\} \\
& \Lambda_{x}=\left\{\xi \in \Sigma_{x} \mid \angle\left(\xi, \gamma_{x}^{+}(0)\right)=\angle\left(\xi, \gamma_{x}^{-}(0)\right)=\pi / 2\right\}
\end{aligned}
$$

Given a sequence $\theta:=\left\{\theta_{j}\right\} \in \mathcal{S}$, we define a function $H_{x}^{\theta} b_{+}: \Lambda_{x} \rightarrow \mathbb{R}$ by

$$
H_{x}^{\theta} b_{+}(\xi) \stackrel{\text { def }}{=} \limsup _{s \rightarrow 0, s \in \theta} \frac{b_{+} \circ \exp _{x}(s \cdot \xi)-b_{+}(x)}{s^{2} / 2}
$$

and

$$
\Delta^{\theta} b_{+}(x) \stackrel{\text { def }}{=}(n-1) \cdot \oint_{\Lambda_{x}} H_{x}^{\theta} b_{+}(\xi)
$$

In the following Lemma 6.3 , we will prove that both $b_{+}$and $b_{-}$are semiconcave. Thus, by Lemma $6.1, H_{x}^{\theta} b_{+}$is well defined and is locally bounded. It is easy to see that $H_{x}^{\theta} b_{+}$is measurable, so $\Delta^{\theta} b_{+}(x)$ is also well defined.

Lemma 6.3. $b_{+}(x)$ is a semi-concave function in $M$. Moreover, for any point $x \in M$ and any sequence $\theta=\left\{\theta_{j}\right\} \in \mathcal{S}$, there exists a subsequence $\delta \subset \theta$ such that $\Delta^{\delta} b_{+}(x) \leqslant 0$. 
Proof. Fix a point $x \in M$, we will construct a semi-concave support function for $b_{+}$near $x$.

We take the line $\gamma_{x}$ in Lemma 6.2 and choose a point $p \in \gamma_{x}$ such that $b_{+}(p) \ll b_{+}(x)$.

The equation $\left(b_{+} \circ \gamma_{x}\right)^{\prime}=1$ implies

$$
b_{+}(x)-b_{+}(p)=|p x| .
$$

On the other hand, since $b_{+}$is 1-Lipschitz, we have

$$
b_{+}(y)-b_{+}(p) \leqslant|p y|
$$

for any $y \in M$. By combining (6.3) and (6.4), we know that function $\operatorname{dist}_{p}(\cdot)$ $+b_{+}(p)$ supports $b_{+}$near $x$.

This tells us $b_{+}$is a semi-concave function. Furthermore, from Theorem 3.1, we can find a subsequence $\widetilde{\delta} \subset \theta$ such that $\Delta^{\widetilde{\delta}} b_{+}(x) \leqslant(n-1) /|p x|$. By letting $|p x| \rightarrow \infty$ and a diagonal argument, we can choose a subsequence $\delta \subset \widetilde{\delta}$ such that $\Delta^{\delta} b_{+}(x) \leqslant 0$. Therefore the proof of the lemma is completed.

The following lemma is similar to Lemma 4.2.

Lemma 6.4. Assume that for each point $x \in M$, there exists a sequence $\theta:=\left\{\theta_{j}\right\} \in \mathcal{S}$ such that $\Delta^{\theta^{\prime}} b_{+}(x)=0$ for any subsequence $\theta^{\prime} \subset \theta$. Then $b_{+}$ is a concave function in $M$.

Proof. It suffices to show that $b_{+}$is concave on an arbitrarily given bounded open set $\Omega \subset M$. Clearly, we may assume $M$ has curvature $\geqslant k_{\Omega}$ in $\Omega$ for some constant $k_{\Omega}$.

In the following, we divide the proof into three steps.

Step 1. Let $\gamma_{x}$ be the line in Lemma 6.2. Replacing Equations (3.6) and (3.7) by the facts that $\left|b_{+}(y)-b_{+}(z)\right|=|y z|$ for any $y, z \in \gamma_{x}$ and $b_{+}$is 1Lipschitz, the same proof in Lemma 3.1 shows that the lemma also holds when we replace $f=\operatorname{dist}_{p}$ by $b_{+}$.

Step 2. Similar as Lemma 4.1, we want to show $H_{x}^{\delta} b_{+}=0$ almost everywhere in $\Lambda_{x}$, for some subsequence $\delta=\left\{\delta_{j}\right\} \subset \theta$.

We now follow the proof of Lemma 4.1. Firstly, from Lemma 6.3, we know that both $b_{+}$and $b_{-}$are semi-concave. In turn, Lemma 6.1 gives a bound for $H_{x}^{\theta} b_{+}$. Secondly, we use Lemma 3.1 for $b_{+}$(i.e., the above Step 1) and replace Theorem 3.1 by the above Lemma 6.3 in the proof of Lemma 4.1. 
We repeat the same proof of Lemma 4.1 to get $H_{x}^{\delta} b_{+}=0$ almost everywhere in $\Lambda_{x}$, for some subsequence $\delta \subset \theta$.

Step 3. Following the proof of Lemma 4.2, we then deduce that $b_{+}(x)$ is concave in $\Omega$. Therefore $b_{+}(x)$ is concave in $M$ and the proof of the lemma is completed.

Now, we are in a position to prove Theorem 1.3.

Proof of Theorem 1.3. Given a sequence $\theta=\left\{\theta_{j}\right\} \in \mathcal{S}$, from Lemma 6.3, we can find a subsequence $\delta \subset \theta$ such that

$$
\Delta^{\delta} b_{+}(x) \leqslant 0 \quad \text { and } \quad \Delta^{\delta} b_{-}(x) \leqslant 0 .
$$

By the definition of $\Delta^{\delta} b_{+}(x)$ and $\Delta^{\delta} b_{-}(x)$, we have

$$
\Delta^{\delta^{\prime}} b_{+}(x) \leqslant \Delta^{\delta} b_{+}(x) \quad \text { and } \quad \Delta^{\delta^{\prime}} b_{-}(x) \leqslant \Delta^{\delta} b_{-}(x)
$$

for any subsequence $\delta^{\prime} \subset \delta$. So (6.5) holds for any subsequence $\delta^{\prime} \subset \delta$.

On the other hand, by Lemma 6.1 and the definition of $\Delta^{\theta} b_{+}(x)$, we have

$$
\Delta^{\vartheta} b_{+}(x)+\Delta^{\vartheta} b_{-}(x) \geqslant 0
$$

for any sequence $\vartheta=\left\{\vartheta_{j}\right\} \in \mathcal{S}$. Therefore, by combining with (6.5), we get

$$
\Delta^{\delta^{\prime}} b_{+}(x)=0 \quad \text { and } \quad \Delta^{\delta^{\prime}} b_{-}(x)=0
$$

for any subsequence $\delta^{\prime} \subset \delta$.

Then we can apply Lemma 6.4 to conclude that both $b_{+}$and $b_{-}$are concave. By using Lemma 6.1 again, we deduce that $b_{+} \circ \varsigma(s)$ is a linear function on any geodesic $\varsigma(s)$ in $M$. In particular, the level surfaces $\mathcal{L}(a):=$ $b_{+}^{-1}(a)$ are totally geodesic for all $a \in \mathbb{R}$.

Set $N=\mathcal{L}(0)=b_{+}^{-1}(0)$. It is an Alexandrov space with curvature bounded below locally.

When $M$ is an Alexandrov space with curvature $\geqslant-\kappa^{2}$ for some $\kappa>0$. Mashiko, in [18], proved that if there exists a function $u$ such that $u \circ \gamma$ is a linear function for any geodesic $\gamma \subset M$ and $u \in D^{2,2}$ (see [18] for the definition of the class of $D^{2,2}$ ), then $M$ is isometric to a direct product $\mathbb{R} \times Y$ over an Alexandrov space $Y$ has curvature $\geqslant-\kappa^{2}$. Later in [2], Alexander and Bishop removed the condition $u \in D^{2,2}$.

Since we do not assume that $M$ has a uniform lower curvature bound, we adapt Mashiko's argument as follows. 
For any $x \in N$ and any $a \in \mathbb{R}$, let $\gamma_{x}$ be the line obtained in Lemma 6.2.

Note that $\left(b_{+} \circ \gamma_{x}\right)(s)^{\prime}=1$ which implies $\nabla b_{+}\left(\gamma_{x}(s)\right)=\gamma_{x}^{+}(s)$. Thus $\gamma_{x}$ is a gradient curve of $b_{+}$.

It is easy to check that $\gamma_{x} \cap \mathcal{L}(a)$ is a set of single point. We define $\Phi_{a}: N \rightarrow \mathcal{L}(a)$ by $\Phi_{a}(x)=\gamma_{x} \cap \mathcal{L}(a)$. $\Phi_{a}$ and $\Phi_{a}^{-1}$ are the gradient flows of $b_{+}$and $b_{-}$, respectively. Since a gradient flow of a concave function is non-expanding, we have that $\Phi_{a}$ is an isometry.

Now we are ready to show that $M$ is isometric to the direct product $\mathbb{R} \times N$. Consider any two points $x, y \in M$.

Without loss of generality, we may assume that $x \in N$ and $y \in \mathcal{L}(a)$ with $a>0$. Let $z=\gamma_{y} \cap N$, where $\gamma_{y}$ comes from Lemma 6.2.

We take a $C^{1}$ curve $\sigma(s) \subset N$ with $\sigma(0)=x$ and $\sigma(\operatorname{Length}(\sigma))=z$, $\left|\sigma^{\prime}(s)\right|=1$. Define a new curve $\bar{\sigma}(s)$ by

$$
\bar{\sigma}(s)=\gamma_{\sigma(s)}\left(\frac{a}{\operatorname{Length}(\sigma)} \cdot s\right) .
$$

Clearly, we have $\bar{\sigma}(0)=x, \bar{\sigma}(\operatorname{Length}(\sigma))=\gamma_{z}(a)=y$ and

$$
b_{+}(\bar{\sigma}(s))=\frac{a}{\operatorname{Length}(\sigma)} \cdot s .
$$

Fixed any $s \in(0$, Length $(\sigma))$, we set $u=\sigma(s)$ and $v=\bar{\sigma}(s)$.

We claim that

$$
\angle\left(\nabla_{u} b_{+}, \sigma^{+}(s)\right)=\angle\left(\uparrow_{u}^{v}, \sigma^{+}(s)\right)=\pi / 2 .
$$

Indeed,

$$
\left|v \sigma\left(s^{\prime}\right)\right| \geqslant b_{+}(v)-b_{+}\left(\sigma\left(s^{\prime}\right)\right)=b_{+}(v)=|v u|
$$

for any $s^{\prime} \in(0$, Length $(\sigma))$. Then by the first variation formula of arc-length, we have

$$
\angle\left(\uparrow_{u}^{v}, \sigma^{+}(s)\right) \geqslant \pi / 2 \text { and } \angle\left(\uparrow_{u}^{v}, \sigma^{-}(s)\right) \geqslant \pi / 2 .
$$

On the other hand,

$$
\angle\left(\uparrow_{u}^{v}, \sigma^{+}(s)\right)+\angle\left(\uparrow_{u}^{v}, \sigma^{-}(s)\right)=\pi .
$$

Thus the desired (6.7) follows from (6.8) and (6.9).

Now let us calculate the length of the curve $\bar{\sigma}$. 


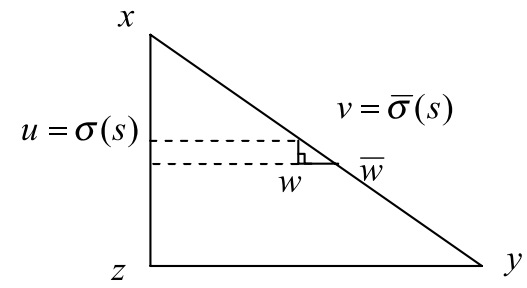

Figure 1: Isometric splitting.

Clearly, we may assume that a neighborhood of $\bar{\sigma}$ has curvature $\geqslant k$ (for some $k<0)$.

Fixed $s \in(0, \operatorname{Length}(\sigma))$. Let $h>0$ be a small number. We set $\bar{w}=$ $\bar{\sigma}(s+h)$ and $w=\gamma_{\sigma(s+h)}\left(\frac{a}{\operatorname{Length}(\sigma)} \cdot s\right)$ (see figure 1).

By cosine law in 0 -plane $\mathbb{R}^{2}$, we have

$$
|\bar{\sigma}(s+h) \bar{\sigma}(s)|^{2}=|v \bar{w}|^{2}=|v w|^{2}+|w \bar{w}|^{2}-2|w \bar{w}| \cdot|v w| \cdot \cos \widetilde{\measuredangle}_{0} v w \bar{w} .
$$

Note that

$$
\begin{aligned}
& |v \bar{w}|=|\sigma(s) \sigma(s+h)|=\left|\sigma^{+}(s) \cdot h+o(h)\right|=h+o(h), \\
& |w \bar{w}|=\left(b_{+}(\bar{w})-b_{+}(w)\right)=\frac{a}{\operatorname{Length}(\sigma)} \cdot h .
\end{aligned}
$$

By using Lemma 11.2 in [4], we have

$$
\widetilde{\angle}_{k} v w \bar{w} \rightarrow \angle v w \bar{w}=\pi / 2
$$

as $h \rightarrow 0$. On the other hand, note that

$$
\widetilde{\measuredangle}_{0} v w \bar{w}-\widetilde{\angle}_{k} v w \bar{w} \rightarrow 0
$$

as $h \rightarrow 0$. We have $\cos \widetilde{\angle}_{0} v w \bar{w} \rightarrow 0$ as $h \rightarrow 0$.

Combining this and (6.10)-(6.12), we have

$$
|\bar{\sigma}(s+h) \bar{\sigma}(s)|^{2}=\left(1+\left(\frac{a}{\operatorname{Length}(\sigma)}\right)^{2}\right) \cdot h^{2}+o\left(h^{2}\right) .
$$


Hence,

$$
|\bar{\sigma}(s)|^{+}=\left(1+\left(\frac{a}{\operatorname{Length}(\sigma)}\right)^{2}\right)^{1 / 2}
$$

Similarly, we can get

$$
|\bar{\sigma}(s)|^{-}=\left(1+\left(\frac{a}{\operatorname{Length}(\sigma)}\right)^{2}\right)^{1 / 2} .
$$

So

$$
\operatorname{Length}(\bar{\sigma})=\int_{0}^{\operatorname{length}(\sigma)}|\bar{\sigma}|^{\prime} d s=\left(a^{2}+(\operatorname{Length}(\sigma))^{2}\right)^{1 / 2}
$$

If we take $\sigma_{1}$ to be a geodesic $x z$, we get, from (6.16), that

$$
|x y|^{2} \leqslant\left(\operatorname{Length}\left(\bar{\sigma}_{1}\right)\right)^{2}=|x z|^{2}+a^{2}=|x z|^{2}+|y z|^{2} .
$$

While if we take $\sigma_{2}$ to be the projection of a geodesic $x y$ to $N$, we get, from (6.16), that

$$
|x y|^{2}=\left(\operatorname{Length}\left(\sigma_{2}\right)\right)^{2}+a^{2} \geqslant|x z|^{2}+|y z|^{2} .
$$

The combination of (6.17) and (6.18) implies that

$$
|x y|^{2}=|x z|^{2}+|y z|^{2} .
$$

This says that $M$ is isometric to the direct product $N \times \mathbb{R}$.

Lastly, we need prove that $N$ has non-negative Ricci curvature.

Let $\gamma(t):(-\ell, \ell) \rightarrow N$ be a geodesic in $N$. Assume that $N$ has curvature $\geqslant K$ in a neighborhood of $\gamma$ and for some $K<0$. Otherwise, there is nothing to prove. Hence $M$ has curvature $\geqslant K$ in a neighborhood of $\gamma$ in $M$.

Let $p$ and $q$ be two interior points in $\gamma$. We denote the tangent spaces, exponential map in $N$ (or $M$, resp.) by $T_{p} N$, $\exp _{p}^{N}$ (or $T_{p} M=T_{(p, 0)} M$, $\exp _{p}^{M}=\exp _{(p, 0)}^{M}$, resp.) and

$$
\Lambda_{p}^{N}=\left\{\xi \in T_{p}^{N}:\left\langle\xi, \gamma^{\prime}\right\rangle=0\right\}
$$

Let $\Lambda_{p}^{M}:=\Lambda_{(p, 0)}^{M}=\left\{\xi \in T_{p}^{M}:\left\langle\xi, \gamma^{\prime}\right\rangle=0\right\}$. Then $\Lambda_{p}^{M}=S\left(\Lambda_{p}^{N}\right)$ with vertex $\zeta^{ \pm}$, where $\zeta^{ \pm}$are the directions along factor $\mathbb{R}$ in $M=N \times \mathbb{R}$. For any 
$\xi \in T_{p} N$, we have

$$
\exp _{p}^{M}(\xi, t)=\left(\exp _{p}^{N}(\xi), t\right)
$$

Suppose that a family of continuous functions $\left\{g_{(\gamma(t), 0)}(\xi, \eta)\right\}_{-\ell<t<\ell}$ on $\Lambda_{p}^{M}=S\left(\Lambda_{p}^{N}\right)$ satisfies Condition (RC) on geodesic $(\gamma(t), 0)$ in $M$ and

$$
\int_{\Lambda_{p}^{M}} g_{(\gamma(t), 0)}(\xi, \eta) d \operatorname{vol}_{\Lambda_{p}^{M}} \geqslant-\epsilon
$$

for a given small number $\epsilon>0$.

Given a sequence $\left\{\widetilde{s}_{j}\right\} \in \mathcal{S}$, the isometry $T: L_{(p, 0)}^{M} \rightarrow L_{(q, 0)}^{M}$ and subsequence $\left\{s_{j}\right\} \subset\left\{\widetilde{s}_{j}\right\}$ come from the definition of Condition (RC). Recall Petrunin's construction for $T$, we can assume that $T\left(\zeta^{+}\right)=\zeta^{+}$, hence $T$ : $L_{p}^{N} \subset T_{p}^{N} \rightarrow L_{q}^{N} \subset T_{q}^{N}$

Given a quasi-geodesic $\sigma(s)$ in $N$, setting $\bar{\sigma}(s)=(\sigma(a s), b s)$ for any two number $a, b \in \mathbb{R}$ with $a^{2}+b^{2}=1$, we will prove that $\bar{\sigma}(s)$ is a quasi-geodesic in $M$.

Let $u(z, r)$ be a $\lambda$-concave function, defined in a neighborhood of $\gamma$ in $M=N \times \mathbb{R}$. So function $u(\cdot, r)$ is $\lambda$-concave in $N$ and $u(z, \cdot)$ is $\lambda$-concave in $\mathbb{R}$ for all $r \in \mathbb{R}$ and $z \in N$. Since $\sigma$ is quasi-geodesic in $N$, we have

$$
u^{\prime \prime}(\sigma(a s), r) \leqslant a^{2} \cdot \lambda
$$

for all $r \in \mathbb{R}$. Now

$$
u^{\prime \prime}(\sigma(a s), b s) \leqslant\left(a^{2}+b^{2}\right) \cdot \lambda=\lambda .
$$

By definition of quasi-geodesic [32], we get that $\bar{\sigma}(s)$ is a quasi-geodesic in $M$.

Fix any non-negative number $l_{1}$ and $l_{2}$. Let $\xi \in \Lambda_{p}^{N}$. For any constant $A \in \mathbb{R}$, we have (see figure 2)

$$
\begin{aligned}
& \left|\exp _{p}^{N}\left(s_{j} \cdot l_{1} \xi\right), \exp _{q}^{N}\left(s_{j} \cdot l_{2} T \xi\right)\right|^{2} \\
& =\left|\left(\exp _{p}^{N}\left(s_{j} \cdot l_{1} \xi\right), s_{j} l_{1} A \zeta^{+}\right),\left(\exp _{q}^{N}\left(s_{j} \cdot l_{2} T \xi\right), s_{j} l_{2} A \zeta^{+}\right)\right|^{2}-A^{2}\left(l_{1}-l_{2}\right)^{2} \cdot s_{j}^{2} \\
& =\mid \exp _{p}^{M}\left(s_{j} \cdot l_{1}\left(\xi, A \zeta^{+}\right)\right),\left(\left.\exp _{q}^{M}\left(s_{j} \cdot l_{2}\left(T \xi, A \zeta^{+}\right)\right)\right|^{2}-A^{2}\left(l_{1}-l_{2}\right)^{2} \cdot s_{j}^{2}\right. \\
& \leqslant|p q|^{2}+s_{j}^{2} \cdot\left(\left(l_{1}-l_{2}\right)^{2}-\frac{g_{p}\left(\xi, A \zeta^{+}\right) \cdot\left(1+A^{2}\right)}{3}|p q|^{2}\left(l_{1}^{2}+l_{1} \cdot l_{2}+l_{2}^{2}\right)\right) \\
& \quad+o\left(s_{j}^{2}\right) .
\end{aligned}
$$




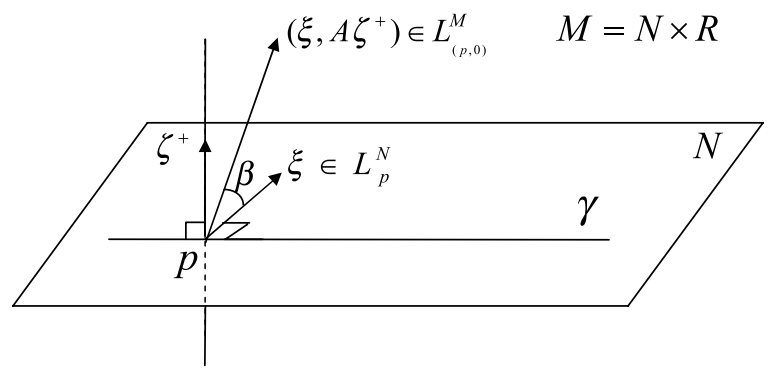

Figure 2: Curvature of factor $N$.

We set $\beta=\angle\left((\xi, 0),\left(\xi, A \zeta^{+}\right)\right)$and then $A=\tan \beta, \beta \in(-\pi / 2, \pi / 2)$. $\mathbb{R}$ by

For each $t \in(-\ell, \ell)$ and $A \in \mathbb{R}$, we define a function $g_{A, \gamma(t)}: \Lambda_{\gamma(t)}^{N} \rightarrow$

$$
\begin{aligned}
g_{A, \gamma(t)}(\xi): & =g_{(\gamma(t), 0)}\left(\xi, A \zeta^{+}\right) \cdot\left(1+A^{2}\right) \\
& =g_{(\gamma(t), 0)}\left(\xi, A \zeta^{+}\right) / \cos ^{2} \beta .
\end{aligned}
$$

From (6.21), for any $A \in \mathbb{R}$, the family of continuous functions $\left\{g_{A, \gamma(t)}\right.$ $(\xi)\}_{-\ell<t<\ell}$ satisfies Condition (RC) on $\gamma$.

On the other hand, we have

$$
\begin{aligned}
-\epsilon & \leqslant \int_{\Lambda_{p}^{M}} g_{(\gamma(t), 0)}(\xi, \eta) d \operatorname{vol}_{\Lambda_{p}^{M}} \\
& =\int_{\Lambda_{p}^{N}} \int_{-\pi / 2}^{\pi / 2} g_{(\gamma(t), 0)}(\xi, \eta) \cos ^{n-2} \beta d \beta d \operatorname{vol}_{\Lambda_{p}^{N}} \\
& =\int_{\Lambda_{p}^{N}} \int_{-\pi / 2}^{\pi / 2} g_{A, \gamma(t)}(\xi) \cos ^{n} \beta d \beta d \operatorname{vol}_{\Lambda_{p}^{N}} \\
& =\int_{-\pi / 2}^{\pi / 2} \int_{\Lambda_{p}^{N}} g_{A, \gamma(t)}(\xi) \cos ^{n} \beta d \operatorname{vol}_{\Lambda_{p}^{N}} d \beta .
\end{aligned}
$$

Thus, we can choose some $A \in \mathbb{R}$ such that

$$
\int_{\Lambda_{p}^{N}}\left(g_{A, \gamma(t)}(\xi)\right) d \operatorname{vol}_{\Lambda_{p}^{N}} \geqslant-c_{n} \cdot \epsilon
$$


for some constant $c_{n}$. This completes the proof that $N$ has non-negative Ricci curvature. Therefore the proof of Theorem 1.3 is completed.

\section{Appendix A}

In the Appendix, we will recall the definition of curvature-dimension condition $\mathrm{CD}(n, k)$ which is given by Sturm [38] and Lott-Villani [16] (see also [41]). After that we will present a proof, due to Petrunin [31], for the statement that an $n$-dimensional Alexandrov space with Ricci curvature $\geqslant(n-1) K$ and with $\partial M=\varnothing$ must satisfy $\operatorname{CD}(n,(n-1) K)$.

Let $(X, d, m)$ be a metric measure space, where $(X, d)$ is a complete separable metric space.

Given two measures $\mu$ and $\nu$ on $X$, a measure $q$ on $X \times X$ is called a coupling (or transference plan) of $\mu$ and $\nu$ if

$$
q(A \times X)=\mu(A) \quad \text { and } \quad q(X \times A)=\nu(A)
$$

for all measurable $A \subset X$.

The $L^{2}$-Wasserstein distance between two measures $\mu, \nu$ is defined by

$$
d_{W}^{2}(\mu, \nu)=\inf _{q} \int_{X \times X} d^{2}(x, y) d q(x, y)
$$

where infimum runs over all coupling $q$ of $\mu$ and $\nu$. If $\mu(X) \neq \nu(X)$, we set $d_{W}(\mu, \nu)=+\infty$.

Let $\mathcal{P}_{2}(X)$ be the space of all probability measures $\nu$ on $X$ with finite second moments:

$$
\int_{X} d^{2}(o, x) d \nu(x)<\infty
$$

for some (hence all) point $o \in X$.

$L^{2}$-Wasserstein space is a complete metric space $\left(\mathcal{P}_{2}(X), d_{W}\right)$. (see [37] for the geometry of $L^{2}$-Wasserstein space.) Fix a Borel measure $m$ on $X$. We denote $L^{2}$-Wasserstein space by $\mathcal{P}_{2}(X, d)$ and its subspace of $m$-absolutely continuous measures is denoted by $\mathcal{P}_{2}(X, d, m)$.

Given $k \in \mathbb{R}, n \in(1, \infty], t \in[0,1]$ and two points $x, y \in X$, we define $\beta_{t}^{(k, n)}$ as follows: 
(1) If $0<t \leqslant 1$, then

$$
\begin{aligned}
& \beta_{t}^{(k, n)}(x, y) \\
& := \begin{cases}\exp \left(\frac{k}{6}\left(1-t^{2}\right) \cdot d^{2}\left(x_{0}, x_{1}\right)\right) & \text { if } n=\infty, \\
\infty & \text { if } n<\infty, k>0 \text { and } \alpha \geqslant \pi, \\
\left(\frac{\sin (t \alpha)}{t \sin \alpha}\right)^{n-1} & \text { if } n<\infty, k>0 \text { and } \alpha \in[0, \pi), \\
1 & \text { if } n<\infty, k=0, \\
\left(\frac{\sinh (t \alpha)}{t \sinh \alpha}\right)^{n-1} & \text { if } n<\infty, k<0,\end{cases}
\end{aligned}
$$

where $\alpha=d(x, y) \cdot \sqrt{|k| /(n-1)}$.

(2) $\beta_{0}^{(k, n)}(x, y)=1$.

The curvature-dimension condition $\operatorname{CD}(n, k)$ is defined as follows (see $[41,29.8,30.32])$ :

Definition A.1. Let $(X, d, m)$ be a non-branching locally compact complete separable geodesic space equipped with a locally finite measure $m .^{3}$

Given two real numbers $k$ and $n$ with $n>1$, the metric measure space $(X, d, m)$ is said to satisfy the curvature-dimension condition $\operatorname{CD}(n, k)$ if and only if for each pair compactly supported $\mu_{0}, \mu_{1} \in \mathcal{P}_{2}(X, d, m)$ there exists an optimal coupling $q$ of $\mu_{0}=\varrho_{0} m$ and $\mu_{1}=\varrho_{1} m$, and a geodesic path ${ }^{4}$ $\mu_{t}:[0,1] \rightarrow \mathcal{P}_{2}(X, d)$ connecting $\mu_{0}$ and $\mu_{1}$, with

$$
\begin{aligned}
H_{n}\left(\mu_{t} \mid m\right) \leqslant & -(1-t) \int_{X \times X}\left(\frac{\varrho_{0}(x)}{\beta_{1-t}^{(k, n)}(x, y)}\right)^{-1 / n} d q(x, y) \\
& -t \int_{X \times X}\left(\frac{\varrho_{1}(y)}{\beta_{t}^{(k, n)}(x, y)}\right)^{-1 / n} d q(x, y)
\end{aligned}
$$

for all $t \in[0,1]$, where $H_{n}(\cdot \mid m): \mathcal{P}_{2}(X, d) \rightarrow \mathbb{R}$ is Rényi entropy functional with respect to $m$,

$$
H_{n}(\mu \mid m):=-\int_{X} \varrho^{-1 / n} d \mu
$$

and $\varrho$ denotes the density of the absolutely continuous part in the Lebesgue decomposition $\mu=\varrho m+\mu^{c}$ of $\mu$.

\footnotetext{
${ }^{3}$ Lott-Villani and Sturm defined curvature dimension condition on general metric measure spaces.

${ }^{4}$ Constant-speed shortest curve defined on $[0,1]$.
} 
From now on, in the Appendix, $M$ will always denote an $n$-dimensional Alexandrov space with $\operatorname{Ric}(M) \geqslant(n-1) K$ and $\partial M=\varnothing$.

Our purpose of this Appendix is to prove the following proposition, which is essentially due to Petrunin [31].

Proposition A.1. Let $M$ be an $n$-dimensional Alexandrov space without boundary and $\operatorname{Ric}(M) \geqslant(n-1) K$. Let vol denote the $n$-dimensional Hausdorff measure on $M$. Then the metric measure space $(M,|\cdot|$, vol) satisfies $\mathrm{CD}(n,(n-1) K)$.

From [38], we know that the curvature-dimension condition $\operatorname{CD}(n,(n-$ 1) $K$ ) implies Bishop-Gromov volume comparison theorem. Consequently, we get the following

Corollary A.1. Let $M$ be as in above proposition. Then the function, for any $p \in M$,

$$
\frac{\operatorname{vol} B_{p}(r)}{\operatorname{vol} B_{K}^{n}(r)}
$$

is non-increasing in $r>0$, where $B_{K}^{n}(r)$ is a geodesic ball of radius $r$ in the $n$-dimensional simply connected Riemannian manifold with constant sectional curvature $K$.

Before beginning the proof of Proposition A.1, let us review some indispensable materials.

For a continuous function $f$, we define its Hamilton-Jacobi shift $\mathcal{H}_{t} f$ for time $t>0$ by

$$
\mathcal{H}_{t} f \stackrel{\text { def }}{=} \inf _{y \in M}\left\{f(y)+\frac{1}{2 t}|x y|^{2}\right\} .
$$

Denote by $f_{t}=\mathcal{H}_{t} f$. A solution of $\alpha^{+}(t)=\nabla_{\alpha(t)} f_{t}$ is called a $f_{t^{-}}$gradient curve.

Refer to [31] for the existence and uniqueness of $f_{t}$-gradient curve and basic propositions of Hamilton-Jacobi shifts. Now we list only facts that is necessary for us to prove Proposition A.1.

Fact $A$ : Let $f: M \rightarrow \mathbb{R}$ be bounded and continuous function and $f_{t}=\mathcal{H}_{t} f$. Assume $\gamma:(0,1) \rightarrow M$ is a $f_{t}$-gradient curve which is also a constant-speed shortest curve. We have 
(i) $f_{t_{1}}(x) \leqslant f_{t_{0}}(y)+\frac{|x y|^{2}}{2\left(t_{1}-t_{0}\right)}$ for any $t_{1}>t_{0}>0$ and $x, y \in M$

(ii) $f_{t_{1}}\left(\gamma\left(t_{1}\right)\right)=f_{t_{0}}\left(\gamma\left(t_{0}\right)\right)+\frac{\left|\gamma\left(t_{0}\right) \gamma\left(t_{1}\right)\right|^{2}}{2\left(t_{1}-t_{0}\right)}$;

(iii) $\nabla f_{t}=\gamma^{+}$and $\left|\nabla f_{t}\right|=\frac{\left|\gamma\left(t_{0}\right) \gamma\left(t_{1}\right)\right|}{t_{1}-t_{0}}=|\gamma(0) \gamma(1)|$.

The following result is a modification of [31, Proposition 2.2], where we replace the condition curvature $\geqslant K$ by the condition $\operatorname{Ric}(M) \geqslant(n-1) K$.

Proposition A.2. Let $M$ be an $n$-dimensional Alexandrov space with Ricci curvature $\geqslant(n-1) K . f: M \rightarrow \mathbb{R}$ be bounded and continuous function and $f_{t}=\mathcal{H}_{t} f$. Assume $\gamma:(0,1) \rightarrow M$ is a $f_{t}$-gradient curve which is also a constant-speed shortest curve. Suppose that the bilinear form $\operatorname{Hess}_{\gamma(t)} f_{t}$ is defined for almost all $t \in(0,1)$.

Then

$$
\begin{aligned}
h_{T}^{\prime} & \leqslant-h_{T}^{2}, \\
h_{V}^{\prime} & \leqslant-(n-1) K|\gamma(0) \gamma(1)|^{2}-\frac{h_{V}^{2}}{n-1}
\end{aligned}
$$

in the sense of distributions, where

$$
h_{T}(t) \stackrel{\text { def }}{=} \operatorname{Hess}_{\gamma(t)} f_{t}\left(\frac{\gamma^{+}}{\left|\gamma^{+}\right|}, \frac{\gamma^{+}}{\left|\gamma^{+}\right|}\right)
$$

and $h_{V}$ is the trace of $\operatorname{Hess}_{\gamma(t)} f_{t}$ in the vertical space $L_{\gamma(t)}$, i.e.,

$$
h_{V}(t) \stackrel{\text { def }}{=} \operatorname{Trace}_{L} \operatorname{Hess}_{\gamma(t)} f_{t} .
$$

Proof. Since the bilinear form $\operatorname{Hess}_{\gamma(t)} f_{t}$ is defined for almost all $t \in(0,1)$, we know from [30] that all $T_{\gamma(t)}, t \in(0,1)$, are isometric to $n$-dimensional Euclidean space. In particular, all $L_{\gamma(t)}, t \in(0,1)$, are isometric to $\mathbb{R}^{n-1}$.

Take two points $0<t_{0}<t_{1}<1$, we may assume that $\operatorname{Hess}_{\gamma(t)} f_{t}$ is defined at $t_{0}$ and $t_{1}$.

Denote by the direction $\xi_{t}=\gamma^{+}(t) /\left|\gamma^{+}(t)\right|, t \in(0,1)$. Then we have

$$
\begin{aligned}
f_{t_{0}}\left(\gamma\left(t_{0}+s\right)\right)= & f_{t_{0}}\left(\gamma\left(t_{0}\right)\right)+s \cdot\left\langle\nabla f_{t_{0}}, \gamma^{+}\left(t_{0}\right)\right\rangle \\
& +\frac{s^{2}}{2} \cdot \operatorname{Hess}_{\gamma\left(t_{0}\right)} f_{t_{0}}\left(\xi_{t_{0}}, \xi_{t_{0}}\right) \cdot\left|\gamma^{+}\left(t_{0}\right)\right|^{2}+o\left(s^{2}\right)
\end{aligned}
$$


and

$$
\begin{aligned}
f_{t_{1}}\left(\gamma\left(t_{1}+l s\right)\right)= & f_{t_{1}}\left(\gamma\left(t_{1}\right)\right)+l s \cdot\left\langle\nabla f_{t_{1}}, \gamma^{+}\left(t_{1}\right)\right\rangle \\
& +\frac{(l s)^{2}}{2} \cdot \operatorname{Hess}_{\gamma\left(t_{1}\right)} f_{t_{1}}\left(\xi_{t_{1}}, \xi_{t_{1}}\right) \cdot\left|\gamma^{+}\left(t_{1}\right)\right|^{2}+o\left(s^{2}\right)
\end{aligned}
$$

for any $l \geqslant 0$. Combining these and the Fact $A$, we get

$$
l^{2} \cdot h_{T}\left(t_{1}\right)-h_{T}\left(t_{0}\right) \leqslant \frac{(l-1)^{2}}{t_{1}-t_{0}}
$$

for any $l \geqslant 0$.

Thus, by choosing $l=\left(1-\left(t_{1}-t_{0}\right) h_{T}\left(t_{1}\right)\right)^{-1} \quad\left(\right.$ when $t_{1}-t_{0}$ suffices small, $1-\left(t_{1}-t_{0}\right) h_{T}\left(t_{1}\right)$ is positive), we get

$$
\frac{h_{T}\left(t_{1}\right)-h_{T}\left(t_{0}\right)}{t_{1}-t_{0}} \leqslant-h_{T}\left(t_{1}\right) \cdot h_{T}\left(t_{0}\right) .
$$

That is,

$$
h_{T}^{\prime} \leqslant-h_{T}^{2}
$$

Fix arbitrary $\epsilon>0$. By our definition of Ricci curvature $\geqslant(n-1) K$ along $\gamma$, there exists a continuous function family $\left\{g_{\gamma(t)}\right\}_{0<t<1} \in \mathcal{F}$ such that

$$
\oint_{\Lambda_{\gamma(t)}} g_{\gamma(t)} \geqslant K-\epsilon, \quad \forall t \in(0,1) .
$$

We may assume $t_{1}-t_{0}$ so small that we can use Equation (1.1) for some isometry $T: \Lambda_{\gamma\left(t_{1}\right)} \rightarrow \Lambda_{\gamma\left(t_{0}\right)}$ and some sequence $\left\{s_{j}\right\} \in \mathcal{S}$.

Given any direction $\eta \in \Lambda_{\gamma\left(t_{1}\right)}$, by setting $\sigma_{0}(s)=\exp _{\gamma\left(t_{0}\right)}(s \cdot T \eta)$ and $\sigma_{1}(s)=\exp _{\gamma\left(t_{1}\right)}(s \eta)$, we know from (1.1) that

$$
\begin{aligned}
\mid \sigma_{0}\left(s_{j}\right) & \left.\sigma_{1}\left(l s_{j}\right)\right|^{2} \leqslant\left|\gamma\left(t_{0}\right) \gamma\left(t_{1}\right)\right|^{2} \\
& +\left((l-1)^{2}-\frac{\left(g_{\gamma\left(t_{1}\right)}-\epsilon\right) \cdot\left|\gamma\left(t_{0}\right) \gamma\left(t_{1}\right)\right|^{2}}{3}\left(l^{2}+l+1\right)\right) \cdot s_{j}^{2}+o\left(s_{j}^{2}\right) .
\end{aligned}
$$

Note that

$$
\begin{aligned}
f_{t_{0}}\left(\sigma_{0}(s)\right) & =f_{t_{0}}\left(\sigma_{0}(0)\right)+\frac{s^{2}}{2} \cdot \operatorname{Hess}_{\gamma\left(t_{0}\right)} f_{t_{0}}(T \eta, T \eta)+o\left(s^{2}\right) \\
f_{t_{1}}\left(\sigma_{1}(l s)\right) & =f_{t_{1}}\left(\sigma_{1}(0)\right)+\frac{(l s)^{2}}{2} \cdot \operatorname{Hess}_{\gamma\left(t_{1}\right)} f_{t_{1}}(\eta, \eta)+o\left(s^{2}\right)
\end{aligned}
$$


for any $l \geqslant 0$. By combining (A.3), (A.4) and Fact A, we get

$$
\begin{aligned}
& l^{2} \cdot \operatorname{Hess}_{\gamma\left(t_{1}\right)} f_{t_{1}}(\eta, \eta)-\operatorname{Hess}_{\gamma\left(t_{0}\right)} f_{t_{0}}(T \eta, T \eta) \\
& \quad \leqslant \frac{(l-1)^{2}}{t_{1}-t_{0}}-\left(t_{1}-t_{0}\right)\left|\gamma^{+}\right|^{2} \cdot\left(g_{\gamma\left(t_{1}\right)}(\eta)-\epsilon\right) \cdot \frac{l^{2}+l+1}{3}
\end{aligned}
$$

for any $l \geqslant 0$. Set $\tau=t_{1}-t_{0}$ and $G=\left|\gamma^{+}\left(t_{1}\right)\right|^{2} \cdot\left(g_{\gamma\left(t_{1}\right)}(\eta)-\epsilon\right)$. By choosing

$$
l=(1 / \tau+\tau G / 6) \cdot\left(1 / \tau-\tau G / 3-\operatorname{Hess}_{\gamma\left(t_{1}\right)} f_{t_{1}}(\eta, \eta)\right)^{-1}
$$

(when $\tau$ suffices small, $1 / \tau-\tau G / 3-\operatorname{Hess}_{\gamma\left(t_{1}\right)} f_{t_{1}}(\eta, \eta)$ and $l$ are positive), we get

$$
\begin{aligned}
& \left(\frac{1}{\tau}-\tau G / 3\right) \cdot\left(\operatorname{Hess}_{\gamma\left(t_{1}\right)} f_{t_{1}}(\eta, \eta)-\operatorname{Hess}_{\gamma\left(t_{0}\right)} f_{t_{0}}(T \eta, T \eta)\right) \\
& \quad \leqslant-\operatorname{Hess}_{\gamma\left(t_{1}\right)} f_{t_{1}}(\eta, \eta) \cdot \operatorname{Hess}_{\gamma\left(t_{0}\right)} f_{t_{0}}(T \eta, T \eta)-G+\tau^{2} G^{2} / 12
\end{aligned}
$$

Note the simple fact that for a bilinear form $\beta(a, a)$ on a $m$-dimensional inner product space $V^{m}$,

$$
\operatorname{trace}_{V^{m}} \beta=\frac{m}{\operatorname{vol}(S)} \int_{S} \beta(a, a) d a
$$

where $S$ is the unit sphere of $V^{m}$ with canonical measure. By taking trace for $\operatorname{Hess}_{\gamma\left(t_{0}\right)} f_{t_{0}}$ ( and $\operatorname{Hess}_{\gamma\left(t_{1}\right)} f_{t_{1}}$ ) in $L_{\gamma\left(t_{0}\right)}$ (and $L_{\gamma\left(t_{1}\right)}$, respectively), we get, from (A.2) and (A.6), that

$$
\begin{aligned}
\frac{h_{V}\left(t_{1}\right)-h_{V}\left(t_{0}\right)}{\tau} \leqslant & -\frac{1}{2(n-1)}\left(h_{V}^{2}\left(t_{0}\right)+h_{V}^{2}\left(t_{1}\right)\right) \\
& -(n-1)(K-2 \epsilon)\left|\gamma^{+}\left(t_{1}\right)\right|^{2}+o(1)
\end{aligned}
$$

when we fix $t_{1}$ and let $t_{0} \rightarrow t_{1}$.

On the other hand, by setting $l=1$ in (A.5) and taking trace, we have

$$
\frac{h_{V}\left(t_{1}\right)-h_{V}\left(t_{0}\right)}{\tau} \leqslant-(n-1)(K-2 \epsilon)\left|\gamma^{+}\left(t_{1}\right)\right|^{2} .
$$

This and (A.7) tell us that $h_{V}$ is locally Lipschitz almost everywhere in $(0,1)$. 
By using (A.7), the arbitrariness of $\epsilon$ and Fact A (iii), we get

$$
h_{V}^{\prime} \leqslant-(n-1) K\left|\nabla f_{t}\right|^{2}-\frac{h_{V}^{2}}{n-1} .
$$

Therefore, we have completed the proof of this proposition.

Now we can follow Petrunin's argument in [31] to prove the above Proposition A.1.

Proof of Proposition A.1. Let $\mu_{0}, \mu_{1} \in \mathcal{P}(M, d, m)$ with compactly supported sets $\operatorname{spt}\left(\mu_{0}\right), \operatorname{spt}\left(\mu_{1}\right)$ and $\mu_{t} \in \mathcal{P}(M, d)$ be a geodesic path. We have

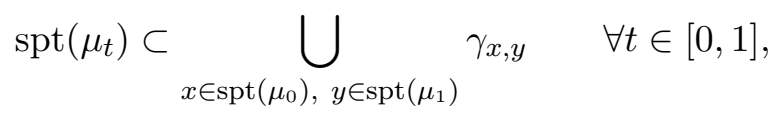

where $\gamma_{x, y}$ is any one geodesic path between $x$ and $y$. Thus we can choose a big enough ball $B$ such that $\operatorname{spt}\left(\mu_{t}\right) \subset B$ for all $t \in[0,1]$. We can find a negative constant $k$ such that $M$ has curvature $\geqslant k$ in $B$.

As shown in $[41,7.22]$, there is a probability measure $\Pi$ on the space of all geodesic paths in $M$ such that if $\Gamma=\operatorname{spt}(\Pi)$ and $e_{t}: \Gamma \rightarrow M$ is evaluation map $e_{t}(\gamma)=\gamma(t)$ then $\mu_{t}=\left(e_{t}\right)_{\#} \Pi$. Let $\Gamma$ be equipped a metric

$$
\left|\gamma \gamma^{\prime}\right|_{\Gamma}:=\max _{t \in[0,1]}\left|\gamma(t) \gamma^{\prime}(t)\right|
$$

According to $[41,5.10]$, there are a pair of optimal price functions $\phi$ and $\psi$ on $M$ such that

$$
\phi(y)-\psi(x) \leqslant \frac{1}{2}|x y|^{2}
$$

for any $x, y \in M$ and equality holds for any $(x, y) \in \operatorname{spt}\left(\left(e_{0}, e_{1}\right)_{\#} \Pi\right)$.

By considering the Hamilton-Jacobi shifts

$$
\psi_{t}=\mathcal{H}_{t} \psi \quad \text { and } \quad \phi_{t}=\mathcal{H}_{1-t}(-\psi)
$$

Petrunin in [31] proved that, for any $t \in(0,1), \mu_{t}$ is absolutely continuous and the evaluation map $e_{t}$ is bi-Lipschitz (where the bi-Lipschitz constant depends on $k$ ). Hence for any measure $\chi$ on $M$, there is uniquely determined one-parameter family of pull-back measures $\chi_{t}^{*}$ on $\Gamma$ such that $\chi_{t}^{*}(E)=$ $\chi\left(e_{t} E\right)$ for any Borel subset $E \subset \Gamma$ (refer to [31] for details).

Fix the measure $\widetilde{\nu}=\operatorname{vol}_{t_{0}=1 / 2}^{*}$ on $\Gamma$. We write $\operatorname{vol}_{t}^{*}=e^{w_{t}} \cdot \widetilde{\nu}$ for some Borel function $w_{t}: \Gamma \rightarrow \mathbb{R}$, since $e_{t}$ is bi-Lipschitz and $v_{t} l_{t}^{*}$ is absolutely continuous with respect to $\widetilde{\nu}$ for any $t \in(0,1)$. 
In [31], Petrunin proved that, for $\Pi-$ a.e. $\gamma \in \Gamma$,

$$
w_{t}=\int_{t_{0}}^{t} \frac{\partial w_{s}}{\partial s} d s \quad \text { a.e. } t \in(0,1)
$$

and

$$
\frac{\partial w_{t}}{\partial t}=h_{t} \quad \text { a.e. } t \in(0,1)
$$

where

$$
h_{t}(\gamma)=\operatorname{TraceHess}_{\gamma(t)} \phi_{t}
$$

Noting that $h_{t}=h_{T}(t)+h_{V}(t)$, we set

$$
w_{t}^{(1)}=\int_{t_{0}}^{t} h_{T}(s) d s, \quad B_{1}(t)=\exp \left(w_{t}^{(1)}\right)
$$

and

$$
w_{t}^{(2)}=\int_{t_{0}}^{t} h_{V}(s) d s, \quad B_{2}(t)=\exp \left(\frac{w_{t}^{(2)}}{n-1}\right) .
$$

By applying Proposition A.2, we get

$$
\begin{aligned}
& B_{1}(t) \geqslant(1-t) B_{1}(0)+t B_{1}(1) \\
& B_{2}(t) \geqslant(1-t) \beta_{1-t}^{1 /(n-1)} B_{2}(0)+t \beta_{t}^{1 /(n-1)} B_{2}(1)
\end{aligned}
$$

where

$$
\beta_{t}=\beta_{t}^{((n-1) K, n)}(\gamma(0), \gamma(1))
$$

Setting $D(t)=\exp \left(w_{t} / n\right)$ and using Hölder inequality

$$
(a+b)^{1 / n} \cdot(c+d)^{(n-1) / n} \geqslant a^{1 / n} \cdot c^{(n-1) / n}+b^{1 / n} \cdot d^{(n-1) / n} \quad \forall a, b, c, d>0,
$$


we have

(A.12)

$$
\begin{aligned}
D(t) & =B_{1}^{1 / n} \cdot B_{2}^{(n-1) / n} \\
& \geqslant\left((1-t) B_{1}(0)+t B_{1}(1)\right)^{\frac{1}{n}} \cdot\left((1-t) \beta_{1-t}^{\frac{1}{n-1}} B_{2}(0)+t \beta_{t}^{\frac{1}{n-1}} B_{2}(1)\right)^{\frac{n-1}{n}} \\
& \geqslant(1-t) \beta_{1-t}^{1 / n} B_{1}(0) B_{2}(0)^{(n-1) / n}+t \beta_{t}^{1 / n} B_{1}(1) B_{2}(1)^{(n-1) / n} \\
& =(1-t) \beta_{1-t}^{1 / n} D(0)+t \beta_{t}^{1 / n} D(1) .
\end{aligned}
$$

Note that Petrunin in [31] had represented $H_{n}\left(\mu_{t} \mid m\right)$ in terms of $w_{t}(\gamma)$ as follows:

$$
H_{n}\left(\mu_{t} \mid m\right)=-\int_{\Gamma} \exp \left(w_{t}(\gamma) / n\right) \cdot a d \Pi
$$

for some non-negative Borel function $a: \Gamma \rightarrow \mathbb{R}$. The combination of this with (A.12) implies the desired inequality (A.1) in the definition of $\mathrm{CD}(n,(n-1) K)$. Therefore we have completed the proof of Proposition A.1.

\section{Acknowledgments}

We would like to thank Dr. Qintao Deng for helpful discussions. We are also grateful to the referee for helpful comments on the second variation formula. The second author is partially supported by NSFC 10831008 and NKBRPC 2006 CB805905.

\section{References}

[1] M. Anderson, On the popology of complete manifolds of non-negative Ricci curvature, Topology 29(1) (1990), 41-55.

[2] S. Alexander and R. Bishop, A cone splitting theorem for Alexandrov spaces, Pacific J. Math. 218 (2005), 1-16.

[3] D. Burago, Y. Burago and S. Ivanov, A course in metric geometry, in 'Graduate Studies in Mathematics', 33, Amer. Math. Soc., Providence, RI, 2001.

[4] Y. Burago, M. Gromov and G. Perelman, A. D. Alexandrov spaces with curvatures bounded below, Russian Math. Surveys 47 (1992), 1-58. 
[5] T. Colding, Large manifolds with positive Ricci curvature, Invent. Math. 124 (1996), 124-193.

[6] J. Cheeger and T. Colding, Lower bounds on Ricci curvature and the almost rigidity of warped products, Ann. Math. 144 (1996), 189-237.

[7] J. Cheeger and T. Colding, On the structure of spaces with Ricci curvature bounded below I, II, III, J. Differential Geom. 46 (1997), 406-480; 54 (2000), 13-35; 54 (2000), 37-74.

[8] J. Cao, B. Dai and J. Mei, An optimal extension of Perelman's comparison theorem for quadrangles and its applications, Recent Adv. Geometric Anal. ALM 11 (2009), 39-59.

[9] J. Cheeger and D. Gromoll, The splitting theorem for manifolds of nonnegative Ricci curvature, J. Differental Geom. 6 (1971), 119-128.

[10] J. Eschenburg and E. Heintze, An elementary proof of the CheegerGromoll splitting theorem, Ann. Global Anal. Geom. 2 (1984), 141-151.

[11] K. Kuwae, Y. Machigashira, T. Shioya, Sobolev spaces, Laplacian and heat kernel on Alexandrov spaces, Math. Z. 238(2) (2001), 269-316.

[12] K. Kuwae and T. Shioya, Laplacian comparison for Alexandrov spaces, http://cn.arxiv.org/abs/0709.0788v1

[13] K. Kuwae and T. Shioya, A topological splitting theorem for weighted Alexandrov spaces, http://cn.arxiv.org/abs/0903.5150v1

[14] K. Kuwae and T. Shioya, On generalized measure contraction property and energy functionals over Lipschitz maps, ICPA98 (Hammamet), Potential Anal. 15(1-2) (2001), 105-121.

[15] K. Kuwae and T. Shioya, Sobolev and Dirichlet spaces over maps between metric spaces, J. Reine Angew. Math. 555 (2003), 39-75.

[16] J. Lott and C. Villani, Ricci curvature for metric-measure spaces via optimal transport, Ann. of Math. 169 (2009), 903-991.

[17] J. Lott and C. Villani, Weak curvature bounds and functional inequalities, J. Funct. Anal. 245(1) (2007), 311-333.

[18] Y. Mashiko, A splitting theorem for Alexandrov spaces, Pacific J. Math. 204 (2002), 445-458. 
[19] A. Milka, Metric structure of some class of spaces containing straight lines, Ukrain. Geometrical. Sbornik, vyp. 4 (1967), 43-48, Kharkov (in Russian).

[20] S. Ohta, On measure contraction property of metric measure spaces, Comment. Math. Helvetici, 82(4) (2007), 805-828.

[21] S. Ohta, Products, cones, and suspensions of spaces with the measure contraction property, J. London Math. Soc. 76(1) (2007), 225-236.

[22] S. Ohta, Finsler interpolation inequalities, Calc. Var. Partial Differential Equations, 36(2) (2009), 211-249.

[23] S. Ohta, Optimal transport and Ricci curvature in Finsler geometry, Adv. Studies Pure Math. 57, Tokyo: Math. Soc. Japan, (2007), 323-342.

[24] Y. Otsu and T. Shioya, The Riemannian structure of Alexandrov spaces, J. Differential Geom. 39 (1994), 629-658.

[25] S. Ohta and K. Sturm, Heat flow on Finsler manifolds, Comm. Pure Appl. Math. 62 (2009), 1386-1433.

[26] Y. Otsu, Differential geometric aspects of Alexandrov spaces, Comparison geometry, (K. Grove and P. Petersen, eds.), M.S.R.I. Publ. 30, Cambridge Univ. Press, (1997), pp. 135-148.

[27] P. Petersen, Riemannian geometry, 2nd Edition, in 'Graduate Texts in Mathematics', 171, Springer, Berlin, 2006.

[28] G. Perelman, A.D.Alexandrov's spaces with curvatures bounded from below, II, Preprint, available online at www.math.psu.edu/petrunin/

[29] G. Perelman, DC structure on Alexandrov spaces, preprint, preliminary version available online at www.math.psu.edu/petrunin/

[30] A. Petrunin, Parallel transportation for Alexandrov spaces with curvature bounded below, Geom. Funct. Anal. 8(1) (1998), 123-148.

[31] A. Petrunin, Alexandrov meets Lott-Villani-Sturm, preprint (2009), available online at www.math.psu.edu/petrunin/

[32] A. Petrunin, Semiconcave functions in Alexandrov's geometry, 'Surveys in Differential Geometry XI: Metric and Comparison Geometry, (2007), pp. 137-201, International Press, Somerville, MA.

[33] A. Petrunin, Harmonic functions on Alexandrov space and its applications, ERA Amer. Math. Soc., 9 (2003), 135-141. 
[34] G. Perelman and A. Petrunin, Quasigeodesics and gradient curves in Alexandrov spaces, preprint, available online at www.math.psu.edu/ petrunin/

[35] M. Renesse, Local Poincare via transportation, Math. Z 259 (2008), 21-31.

[36] M. Renesse and K. Sturm, Transport inequalities, gradient estimates, entropy, and Ricci curvature, Comm. Pure Appl. Math. 58 (2005), 923940.

[37] K. Sturm, On the geometry of metric measure spaces. I. Acta Math. 196(1) (2006), 65-131.

[38] K. Sturm, On the geometry of metric measure spaces. II, Acta Math. 196(1) (2006), 133-177.

[39] K. Sturm, Diffusion processes and heat kernels on metric spaces, Ann. Probab. 26 (1998), 1-55.

[40] K. Sturm, Convex functionals of probability measures and nonlinear diffusions on manifolds, J. Math. Pures Appl. 84(9) (2005), 149-168.

[41] C. Villani, Optimal transport, old and new, in 'Grundlehren der mathematischen Wissenschaften', 338, Springer, Berlin, 2008.

[42] M. Watanabe, Ends of metric measure spaces with nonnegative Ricci curvature, Adv. Studies Pure Math. 55, Tokyo: Math. Soc. Japan, (2006), pp. 335-344.

Department of Mathematics

SUN YAT-SEN UNIVERSITY

GuANGZHOU 510275

P.R. CHINA

E-mail addresses: zhhuich@mail2.sysu.edu.cn; stszxp@mail.sysu.edu.cn

ReCEIVED March 3, 2010 
